\title{
Large gaps and high accretion rates in photoevaporative transition disks with a dead zone
}

\author{
Matías Gárate $^{1,2} \odot$, Timmy N. Delage ${ }^{1} \odot$, Jochen Stadler ${ }^{1} \odot$, Paola Pinilla ${ }^{1,3}$, Til Birnstiel ${ }^{2,4} \odot$, \\ Sebastian Markus Stammler ${ }^{2}$, Giovanni Picogna ${ }^{2} \odot$, Barbara Ercolano $^{2}$, Raphael Franz ${ }^{2}$, and Christian Lenz ${ }^{1}$ \\ ${ }^{1}$ Max-Planck-Institut für Astronomie, Königstuhl 17, 69117 Heidelberg, Germany \\ e-mail: garate@mpia.de \\ ${ }^{2}$ University Observatory, Faculty of Physics, Ludwig-Maximilians-Universität München, Scheinerstr. 1, 81679 Munich, Germany \\ ${ }^{3}$ Mullard Space Science Laboratory, University College London, Holmbury St Mary, Dorking, Surrey RH5 6NT, UK \\ ${ }^{4}$ Exzellenzcluster ORIGINS, Boltzmannstr. 2, 85748 Garching, Germany
}

Received 31 May 2021 / Accepted 6 September 2021

\begin{abstract}
Context. Observations of young stars hosting transition disks show that several of them have high accretion rates, despite their disks presenting extended cavities in their dust component. This represents a challenge for theoretical models, which struggle to reproduce both features simultaneously.

Aims. We aim to explore if a disk evolution model, including a dead zone and disk dispersal by X-ray photoevaporation, can explain the high accretion rates and large gaps (or cavities) measured in transition disks.

Methods. We implemented a dead zone turbulence profile and a photoevaporative mass-loss profile into numerical simulations of gas and dust. We performed a population synthesis study of the gas component and obtained synthetic images and SEDs of the dust component through radiative transfer calculations.

Results. This model results in long-lived inner disks and fast dispersing outer disks that can reproduce both the accretion rates and gap sizes observed in transition disks. For a dead zone of turbulence $\alpha_{\mathrm{dz}}=10^{-4}$ and an extent $r_{\mathrm{dz}}=10 \mathrm{AU}$, our population synthesis study shows that $63 \%$ of our transition disks are still accreting with $\dot{M}_{\mathrm{g}} \geq 10^{-11} M_{\odot} \mathrm{yr}^{-1}$ after opening a gap. Among those accreting transition disks, half display accretion rates higher than $5.0 \times 10^{-10} M_{\odot} \mathrm{yr}^{-1}$. The dust component in these disks is distributed in two regions: in a compact inner disk inside the dead zone, and in a ring at the outer edge of the photoevaporative gap, which can be located between 20 and $100 \mathrm{AU}$. Our radiative transfer calculations show that the disk displays an inner disk and an outer ring in the millimeter continuum, a feature that resembles some of the observed transition disks.

Conclusions. A disk model considering X-ray photoevaporative dispersal in combination with dead zones can explain several of the observed properties in transition disks, including the high accretion rates, the large gaps, and a long-lived inner disk at millimeter emission.
\end{abstract}

Key words. accretion, accretion disks - protoplanetary disks - hydrodynamics - methods: numerical

\section{Introduction}

The nature of the observed structures in protoplanetary disks and their relation to the disk evolution has been a subject of study for over $30 \mathrm{yr}$. Transition disks, in particular, are disks that present a deficit in the near-infrared (NIR) and mid-infrared (MIR) emission, while still displaying the characteristic far-infrared (FIR) excess of most protoplanetary disks (Strom et al. 1989; Skrutskie et al. 1990). They represent one of the key puzzle pieces to understanding the disk evolution process (see reviews by Owen 2016; Ercolano \& Pascucci 2017).

The lack of NIR and MIR emission in transition disks' spectral energy distribution (SED) is attributed to a gap in the dust component, or, more precisely, to the lack of hot micron-sized grains in the inner disk (see Espaillat et al. 2014, for a review). Through radiative transfer models, it has been possible to measure the radial extent of the dust cavity for a wide sample of transition disks (van der Marel et al. 2016b), and observations at different wavelengths have also shown that some of these objects retain a compact dust component close to the star (e.g. Espaillat et al. 2010; Benisty et al. 2010; Olofsson et al. 2013; Matter et al.
2016; Kluska et al. 2018; Pinilla et al. 2019, 2021), demonstrating that for some transition disks the gap is not completely devoid of dust.

One particularly curious and challenging feature of transition disks is that a large number of them show gas accretion signatures, with rates that can be as high as $\dot{M}_{\mathrm{g}} \sim 10^{-8} M_{\odot} \mathrm{yr}^{-1}$ (e.g., Cieza et al. 2012; Alcalá et al. 2014; Manara et al. 2014, 2017). This indicates that there is plenty of gaseous material close to the star, or that low-density material accretes at supersonic speeds into the central star and is driven by a combination of winds and magneto-hydrodynamic (MHD) processes (e.g., Wang \& Goodman 2017). If the inner cavities are really rich in gas, then this raises the following question: how is it possible to create a prominent dust gap while still retaining a long-lived inner gas disk that can produce such accretion rates?

Classical theoretical models suggest that disk evolution occurs in two different stages: a viscous evolution stage, where the transport of angular momentum drives the accretion of gas onto the star (Lynden-Bell \& Pringle 1974; Pringle 1981), and a dispersal stage, where the mass-loss rate due to photoevaporation overcomes the accretion rate onto the star, creating a cavity 
and dispersing the disk from the inside out (Clarke et al. 2001; Alexander et al. 2006a,b; Alexander \& Armitage 2007).

Though photoevaporation models can easily predict large gaps that extend for tens of $\mathrm{AU}$, these fail to explain the observed high accretion rates, since the gaseous inner disks are short lived and quickly accreted once the gap opens (Owen et al. 2010, 2011; Picogna et al. 2019). As a consequence, these models tend to over-predict the fraction of non-accreting disks with gaps that extend beyond $20 \mathrm{AU}$, although the mechanisms that speed up the depletion of the outer disk, such as thermal sweeping and depletion of carbon and oxygen, can partially alleviate the discrepancy with observations (Owen et al. 2013; Ercolano et al. 2018; Wölfer et al. 2019).

On the other hand, planet-disk interactions allow for high accretion rates in the inner disk (Lubow \& D'Angelo 2006), and they also create a gap where the large dust particles are trapped at its outer edge (Pinilla et al. 2012a). Depending on the planet mass and disk properties (e.g. disk viscosity), the inner disk can be replenished with micron-sized particles from the outer disk. However, to date planets have only been detected in the cavity of one transition disk (PDS 70, Keppler et al. 2018; Christiaens et al. 2019), while current observational capabilities should already have detected some of the planets inferred to explain the structures of some of them (e.g. Asensio-Torres et al. 2021). This questions the universality of planets as the potential origin of the transition-disk-like structures. Invoking multiple planets can explain a wider variety of structures (Pinilla et al. 2015), but it can also reduce the lifetime of the inner disk, leading to the same problems as those of photoevaporation models (Zhu et al. 2011).

Other models, including grain growth and dead zones (i.e., regions with low turbulent viscosity, Gammie 1996), show that these can create transition-disk structures (Dullemond \& Dominik 2005; Birnstiel et al. 2012a; Flock et al. 2015) comparable to the ones observed at different wavelengths (e.g., Regály et al. 2012; Pinilla et al. 2016a).

In this work, we aim to explain the accretion rates and gap sizes observed in transition disks by revisiting and expanding the model of Morishima (2012), which studied the evolution of disks with a dead zone undergoing photoevaporative dispersal. In their model, a protoplanetary disk is expected to evolve through the following steps: first, the disk's viscous evolution is driven by the turbulence created by the magnetorotational instability (MRI, Balbus \& Hawley 1998). In the inner regions, the ionization fraction is low, creating a "dead zone" where the MRI is inefficient and the turbulent viscosity is low (Gammie 1996). Then, because of the difference in turbulent viscosity, the inner disk evolves more slowly than the outer disk. This causes the accretion rate at the outer "active" regions to decrease faster than in the inner "dead" regions. Finally, photoevaporation clears a gap outside the dead zone, once the accretion rate has dropped below the mass-loss rate. Meanwhile, the inner disk is unaffected by photoevaporation. This results in a long-lived inner disk that acts as an accretion reservoir, and a large gap in the outer disk that continues to expand from the inside out due to photoevaporation (Morishima 2012; Bae et al. 2013).

The previous study of Morishima (2012) demonstrated that large gaps and high accretion rates can be recreated through the combined effects of dead zones and photoevaporation; however, it is still unclear whether this model can produce a fraction of accreting transition disks consistent with observations (see Hardy et al. 2015; Owen 2016), what the predicted distribution of accretion rates and cavity sizes is, and if the dust component can reproduce the characteristic features of transition disks, such as the deficit in the NIR and MIR emission.
Motivated by these open questions, we constructed a series of population synthesis models, where we included the stateof-the-art X-ray photoevaporation model from Picogna et al. (2019) and a parametric dead-zone prescription, and implement them into the modular disk evolution code DustPy (Stammler $\&$ Birnstiel, in prep.). For each population, we want to measure the gas accretion rate and the gap size distribution, compare it with the observed population of transition disks, and determine which dead-zone properties are more likely to produce high accretion rates with large gaps, without overestimating the fraction of non-accreting disks.

To test if this model can produce a signal that is consistent with observations of transition disks, we also include a dust coagulation model, where we track the evolution of the grain size distribution during disk dispersal, and obtain synthetic SEDs and images at both NIR and millimeter wavelengths.

This paper is organized as follows. In Sect. 2, we present our disk evolution model. In Sect. 3, we describe our numerical setup of our simulations. In Sect. 4, we show the results of our population synthesis study, focusing on the gas evolution. Section 5 shows the evolution of a single simulation including the evolution of dust, along with synthetic images from a radiative transfer calculation. In Sect. 6, we discuss how our model relates to the observed population of transition disks, its caveats, and follow-up work. We summarize our results in Sect. 7.

\section{Disk evolution model}

For our model, we considered an axisymmetric protoplanetary disk undergoing viscous accretion, and we describe its gas surface-density evolution in 1D using the following diffusion equation (Lüst 1952; Lynden-Bell \& Pringle 1974; Pringle 1981):

$\frac{\partial}{\partial t} \Sigma_{\mathrm{g}}=\frac{3}{r} \frac{\partial}{\partial r}\left(r^{1 / 2} \frac{\partial}{\partial r}\left(v \Sigma_{\mathrm{g}} r^{1 / 2}\right)\right)-\dot{\Sigma}_{\mathrm{w}}$,

where $\Sigma_{\mathrm{g}}$ is the gas surface density, $r$ is the radial distance to the star (in cylindrical coordinates), $v$ is the kinematic viscosity, and $\dot{\Sigma}_{\mathrm{w}}$ is the mass-loss rate due to photoevaporation.

For the kinematic viscosity, we adopted the Shakura \& Sunyaev (1973) model:

$v=\alpha c_{\mathrm{s}} h_{\mathrm{g}}$,

where $\alpha$ is a dimensionless parameter that controls the intensity of the disk turbulence, $h_{\mathrm{g}}$ is the gas scale height, and $c_{\mathrm{s}}=$ $\sqrt{\gamma k_{\mathrm{B}} T / \mu m_{\mathrm{p}}}$ is the sound speed, with $k_{\mathrm{B}}$ the Boltzmann constant, $T$ the disk temperature, $\mu=2.3$ the mean molecular weight, $m_{\mathrm{p}}$ the proton mass, and $\gamma=1.4$ the adiabatic index.

In terms of advection, the radial accretion rate of a gas disk is $\dot{M}_{\mathrm{g}}=2 \pi r \Sigma_{\mathrm{g}} v_{\mathrm{g}}$, where $v_{\mathrm{g}}$ corresponds to the gas viscous velocity:

$v_{\mathrm{g}}=-3 \alpha c_{\mathrm{s}} \frac{h_{\mathrm{g}}}{r} \frac{\mathrm{d} \ln }{\mathrm{d} \ln r}\left(v \Sigma_{\mathrm{g}} \sqrt{r}\right)$.

Since the viscous evolution is controlled by the $\alpha$ parameter, we can already infer that regions with low turbulence evolve slowly, and regions with high turbulence evolve rapidly.

\subsection{Photoevaporation model}

Photoevaporation is believed to play a major role in the dispersal of protoplanetary disks. As the accretion rate decreases over 


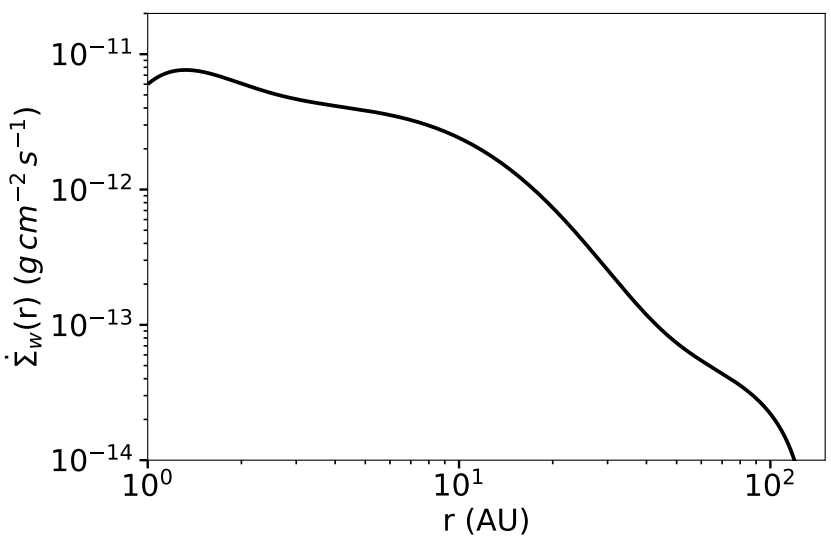

Fig. 1. Surface density loss rate due to X-ray photoevaporation, for a star with $L_{x}=10^{30} \mathrm{erg} \mathrm{s}^{-1}$, following Picogna et al. (2019).

time due to viscous evolution, disks naturally transition to a photoevaporating regime where a gap opens from inside out, once the mass-loss rate surpasses the local gas accretion rate (Clarke et al. 2001; Alexander et al. 2006a,b).

For this study, we focused on the effects of X-ray photoevaporation (Ercolano et al. 2008, 2009; Owen et al. 2010) and implemented the mass-loss rate profiles from Picogna et al. (2019) into the sink term $\dot{\Sigma}_{\mathrm{w}}$ of Eq. (1).

We refer the reader to the original Picogna et al. (2019, their Eqs. (2)-(5)) paper for a detailed description of the mass-loss rate, which is derived from $2 \mathrm{D}$ hydrodynamic models with radiative transfer calculations and parameterized as a function of the stellar X-ray luminosity $L_{x}$. Figure 1 shows the corresponding $\dot{\Sigma}_{\mathrm{w}}$ profile used for this work.

For reference, in this model an X-ray luminosity of $L_{x}=$ $10^{30} \mathrm{erg} \mathrm{s}^{-1}$ corresponds to a total mass-loss rate of $\dot{M}_{\mathrm{w}} \approx$ $10^{-8} M_{\odot} \mathrm{yr}^{-1}$. Furthermore, it is overall higher than the previous photoevaporative model from Owen et al. (2010), making it easier to open a large gap in the gas.

\subsection{Dead-zone model}

Turbulence driven by the magneto rotational instability (MRI) is one of the candidates to explain the angular momentum transport across protoplanetary disks (Balbus \& Hawley 1998), and it is triggered by the coupling between the charged particles in the gas phase and the magnetic field (Balbus \& Hawley 1991). In the inner regions of the disk, where the column density is higher and the ionization lower, the MRI is quenched or even shut off, creating a dead zone (Gammie 1996). In contrast with the MRI active regions, the turbulence in the dead zone is much lower and is dominated by less efficient hydrodynamic instabilities, such as the vertical shear instability (VSI, Nelson et al. 2013; Flock et al. 2020; Manger et al. 2020).

In our $1 \mathrm{D}$ model, we parameterized the turbulence across the protoplanetary disk using the following profile:

$\alpha(r)=\alpha_{\mathrm{dz}}+\left(\alpha_{\mathrm{a}}-\alpha_{\mathrm{dz}}\right) \cdot \begin{cases}\frac{1}{2} \exp (\Delta r / w) & r<r_{\mathrm{dz}} \\ 1-\frac{1}{2} \exp (-\Delta r / w) & r \geq r_{\mathrm{dz}},\end{cases}$

where $\alpha_{\mathrm{a}}$ is the turbulence parameter of the MRI active region, $\alpha_{\mathrm{dz}}$ is the turbulence parameter of the dead zone, $r_{\mathrm{dz}}$ is the deadzone radial extent, $\Delta r=r-r_{\mathrm{dz}}$, and $w=r_{\mathrm{dz}} / 5$ is the transition width from between the dead and the active regions; this is similar to the models used by Birnstiel et al. (2012a), and Gárate et al. (2019). For reference, we show the profile in Fig. 2. This profile

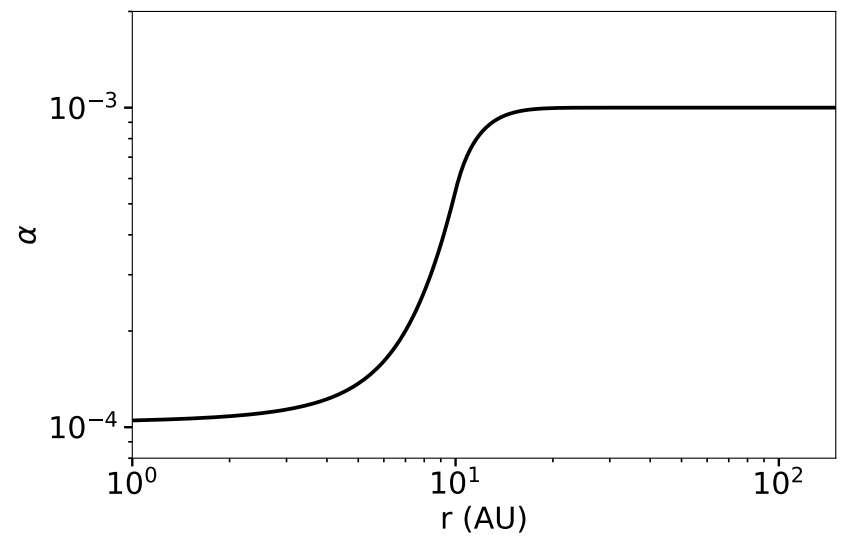

Fig. 2. $\alpha$-parameter radial profile from Eq. (4), using $\alpha_{\mathrm{a}}=10^{-3}, \alpha_{\mathrm{dz}}=$ $10^{-4}$, and $r_{\mathrm{dz}}=10 \mathrm{AU}$.

results in a fast viscous evolution for the outer disk $\left(r>r_{\mathrm{dz}}\right)$ and a slow viscous evolution for the dead zone in the inner disk $\left(r<r_{\mathrm{dz}}\right)$.

While this profile does not include the dependence on the surface density profile from other models (e.g., Kretke et al. 2009; Morishima 2012; Pinilla et al. 2016a), it makes it easier to study the impact of the dead zone extent on the population of accreting transition disks. We discuss this point in Sect. 6.3 and compare our approach with recent dead-zone models. Other mechanism angular momentum transport mechanisms, such as magnetic winds (Blandford \& Payne 1982), could lead to different disk accretion profiles, and it might be worth considering their effect on the global gas surface-density evolution for future studies (Suzuki et al. 2016).

\subsection{Dust evolution}

Since we want to include a radiative transfer calculation in this work, we also need to include a model for the dust dynamics, since these differ from the dynamics of the gas (Whipple 1972; Weidenschilling 1977), as revealed by observations of disks at different wavelengths (e.g., TW Hya, Andrews et al. 2016; van Boekel et al. 2017; Huang et al. 2018). While small particles are indeed well coupled to the gas motion, larger particles can decouple from it, depending on their size. A useful quantity to characterize the level of coupling is the Stokes number, $\mathrm{St}=$ $t_{\text {stop }} \Omega_{\mathrm{k}}$, where $t_{\text {stop }}$ is the time necessary for the dust grains to couple to the gas motion due to their mutual drag force, and $\Omega_{\mathrm{k}}$ is the Keplerian angular velocity.

For spherical dust grains located at the disk midplane, we can write the Stokes number of a particle of radius $a$ and material density $\rho_{\mathrm{s}}$ as

$\mathrm{St}=\frac{\pi}{2} \frac{a \rho_{\mathrm{s}}}{\Sigma_{\mathrm{g}}} \cdot \begin{cases}1 & \lambda_{\mathrm{mfp}} / a \geq 4 / 9 \\ \frac{4}{9} \frac{a}{\lambda_{\mathrm{mfp}}} & \lambda_{\mathrm{mfp}} / a<4 / 9\end{cases}$

where $\lambda_{\mathrm{mfp}}=\left(n \sigma_{\mathrm{H}_{2}}\right)^{-1}$ is the mean free path, $n$ the number density, and $\sigma_{\mathrm{H}_{2}}=2 \times 10^{-15} \mathrm{~cm}^{2}$ the molecular cross-section.

Because most of the mass is concentrated in large grains, and these tend to settle toward the midplane, Eq. (5) is a convenient expression to describe the dust aerodynamic behavior.

Following Nakagawa et al. (1986) and Takeuchi \& Lin (2002), the dust radial velocity is given by

$v_{\mathrm{d}}=\frac{1}{1+\mathrm{St}^{2}} v_{\mathrm{g}}-\frac{2 \mathrm{St}}{1+\mathrm{St}^{2}} \eta v_{\mathrm{k}}$, 
with $\eta=-(1 / 2)\left(h_{\mathrm{g}} / r\right)^{2} \mathrm{~d} \ln P / \mathrm{d} \ln r, v_{\mathrm{k}}$ the Keplerian orbital velocity, $P=\rho_{\mathrm{g}, 0} c_{\mathrm{s}}^{2} / \gamma$ the isothermal pressure, $h_{\mathrm{g}}=c_{\mathrm{s}} / \Omega_{\mathrm{k}}$ the gas scale height, and $\rho_{\mathrm{g}, 0}$ the gas volume density at the midplane.

It can also be useful to think of the Stokes number as a "dynamical grain size". From Eq. (6), we see that small grains (with $\mathrm{St} \ll 1$ ) move with the viscous velocity of the gas, large boulders $(\mathrm{St} \gg 1)$ do not move in the radial direction, and midsized pebbles $(\mathrm{St} \approx 1)$ drift toward the pressure maximum at $v_{\mathrm{d}} \approx-\eta v_{\mathrm{k}}$.

In addition to advection, dust particles also diffuse according to the concentration gradient, with a diffusivity of $D_{\mathrm{d}}=$ $v /\left(1+\mathrm{St}^{2}\right)$ (Youdin \& Lithwick 2007). The dust evolution is then described by the following advection-diffusion equation (Birnstiel et al. 2010):

$$
\frac{\partial}{\partial t}\left(r \Sigma_{\mathrm{d}}\right)+\frac{\partial}{\partial r}\left(r \Sigma_{\mathrm{d}} v_{\mathrm{d}}\right)-\frac{\partial}{\partial r}\left(r D_{\mathrm{d}} \Sigma_{\mathrm{g}} \frac{\partial}{\partial r}\left(\frac{\Sigma_{\mathrm{d}}}{\Sigma_{\mathrm{g}}}\right)\right)=-\dot{\Sigma}_{\mathrm{w}, \mathrm{d}},
$$

where $\Sigma_{\mathrm{d}}$ is the dust surface density (of a particular dust species), and $\dot{\Sigma}_{\mathrm{w}, \mathrm{d}}$ is the dust-loss rate due to entrainment with the photoevaporative wind (Hutchison et al. 2016; Franz et al. 2020; Hutchison \& Clarke 2021; Booth \& Clarke 2021), which we describe below. In this work, we ignored the effects of the dust back-reaction onto the gas dynamics, since these become negligible at low dust-to-gas ratios $(\epsilon \leq 0.01)$ and over timescales longer than 1 Myr (Gárate et al. 2020).

\subsubsection{Dust settling and wind entrainment}

Dust models predict that as particles grow in size, they also tend to settle toward the midplane (Dubrulle et al. 1995), a behavior that is confirmed by observations (see Villenave et al. 2019, 2020, for a prominent example). Assuming that the gas is in hydrostatic equilibrium with a characteristic scale height $h_{\mathrm{g}}$, the dust scale height can be approximated by (Youdin \& Lithwick 2007)

$h_{\mathrm{d}}=h_{\mathrm{g}} \cdot \min \left(1, \sqrt{\frac{\alpha}{\min (\mathrm{St}, 1 / 2)\left(1+\mathrm{St}^{2}\right)}}\right)$.

Then, the vertical structure of the gas and dust can be modeled through a Gaussian distribution (Fromang \& Nelson 2009), with

$\rho_{\mathrm{g}, \mathrm{d}}(z)=\frac{\Sigma_{\mathrm{g}, \mathrm{d}}}{\sqrt{2 \pi} h_{\mathrm{g}, \mathrm{d}}} \exp \left(-\frac{z^{2}}{2 h_{\mathrm{g}, \mathrm{d}}^{2}}\right)$.

Since small particles are well coupled to the gas and we expect the photoevaporative wind to be launched from the disk surface, we can estimate the dust-loss rate due to wind entrainment as

$\dot{\Sigma}_{\mathrm{w}, \mathrm{d}}=\epsilon_{a_{\mathrm{w}}, h_{\mathrm{w}}} \dot{\Sigma}_{\mathrm{w}}$

where $\epsilon_{a_{\mathrm{w}}}, h_{\mathrm{w}}$ is the dust-to-gas ratio of small entrained particles $\left(a \leq a_{\mathrm{w}}=10 \mu \mathrm{m}\right)$ above several gas scale heights $\left(z \geq h_{\mathrm{w}}=\right.$ $\left.3 h_{\mathrm{g}}\right)$. We picked these limits based on the model of Franz et al. (2020), which indicates that only small particles that are several scale heights above the midplane can become entrained with the wind. While this approximation is simplistic, we find it effective because the dependency of the mass-loss rate with the $a_{\mathrm{w}}$ and $h_{\mathrm{w}}$ parameters is very weak (Gárate et al., in prep.).

\subsubsection{Dust growth}

The last ingredients of our dust evolution model are growth and fragmentation, which are necessary if we intend to model the simultaneous evolution of gas and dust (e.g., Brauer et al. 2008; Birnstiel et al. 2010; Dra̧żkowska et al. 2019).

In this work, we considered that the dust phase consists of a distribution of particle species with different sizes, with their dynamics determined by their Stokes number (Eq. (5)), that can also evolve through sticking and fragmentation, as described in Birnstiel et al. (2010). Without entering into the details of the coagulation model, we want to remark that the growth of a particle distribution is locally limited either by drift, when the drift timescale exceeds the growth timescale, or by fragmentation, when the collision velocity between particles exceeds the material fragmentation velocity $v_{\text {frag }}$ (Ormel \& Cuzzi 2007; Brauer et al. 2008; Birnstiel et al. 2009).

Then, the maximum Stokes number that a particle can reach in each case is

$\mathrm{St}_{\mathrm{drift}}=\left|\frac{\mathrm{d} \ln P}{\mathrm{~d} \ln r}\right|^{-1} \frac{v_{\mathrm{k}}^{2}}{c_{\mathrm{s}}^{2}} \epsilon$,
$\mathrm{St}_{\text {frag }}=\frac{1}{3} \frac{v_{\text {frag }}^{2}}{\alpha c_{\mathrm{s}}^{2}}$,

with the maximum grain size given by $\mathrm{St}_{\max }=\min \left(\mathrm{St}_{\mathrm{frag}}, \mathrm{St}_{\mathrm{drift}}\right)$ (Birnstiel et al. 2010, 2012b).

Because the collision velocity between particles depends on the local turbulence parameter (Ormel \& Cuzzi 2007), with

$\Delta v_{\mathrm{turb}} \approx \sqrt{\frac{3 \alpha}{\mathrm{St}+\mathrm{St}^{-1}}} c_{\mathrm{s}}$

we can expect particles to grow into larger sizes in regions with low $\alpha$ (see Eq. (12)), such as the dead zone (Birnstiel et al. 2012a; Pinilla et al. 2016a; Ueda et al. 2019).

\section{Simulation setup}

We performed our numerical simulations using the code DustPy $^{1}$ (Stammler and Birnstiel, in prep.), which can simulate the advection of gas and dust, along with the growth and fragmentation of multiple particle species, following the model of Birnstiel et al. (2010). The code also allows us to load custom modules, which is how we implemented the photoevaporation and dead-zone profiles described in Sect. 2.

This study is separated into "gas-only" simulations, which are fast to run and ideal for population synthesis studies (which are the main focus of this work) and a single "gas-and-dust" simulation, in which we solve the evolution of both components simultaneously to perform a radiative transfer calculation of a specific case in order to compare with multiwavelength observations. In this section, we describe the parameters used to set up the protoplanetary disk, the numerical grid, and the parameter space explored.

\subsection{Disk setup}

For our simulations, we used a solar mass star, and a circumstellar disk with an initial mass of $M_{\text {disk }}=0.1 M_{\odot}$. The initial gas

\footnotetext{
1 A previous version of DustPy was used for this paper. The current
} version is available at github.com/stammler/DustPy 
Table 1. Parameter space for dead-zone models.

\begin{tabular}{cc}
\hline \hline Variable & Value \\
\hline$\alpha_{\mathrm{a}}$ & $10^{-3}$ \\
$\alpha_{\mathrm{dz}}$ & {$[1,3,5] \times 10^{-4}$} \\
$r_{\mathrm{dz}}[\mathrm{AU}]$ & {$[5,10,20]$} \\
\hline
\end{tabular}

surface density is defined with the Lynden-Bell \& Pringle (1974) self-similar solution:

$\Sigma_{\mathrm{g}}(r)=\Sigma_{0}\left(\frac{r}{r_{\mathrm{c}}}\right)^{-1} \exp \left(-r / r_{\mathrm{c}}\right)$.

This initial condition is defined by the characteristic radius $r_{\mathrm{c}}$ at which the exponential drop begins, and a normalization factor $\Sigma_{0}$, defined such that $\int 2 \pi r \Sigma_{\mathrm{g}} \mathrm{d} r=M_{\text {disk }}$.

For the gas temperature, we assumed that the disk is heated passively by the central star, and use the following profile:

$T(r)=150\left(\frac{r}{1 \mathrm{AU}}\right)^{-1 / 2} \mathrm{~K}$.

For all our simulations, the radial grid extends from $1 \mathrm{AU}$ to $300 \mathrm{AU}$, with $n_{\mathrm{r}}=250$ logarithmically spaced grid cells. Additionally, in Appendix A we test the effect of the initial condition on our results; specifically, we present what would happen if the disk inner regions were in a quasi-steady state from the beginning of the simulation (i.e., a radially constant accretion profile that decreases over time).

\subsection{Population synthesis}

In this work, we constructed ten different disk populations, consisting of one "control" population without a dead zone (i.e., $\alpha(r)=\alpha_{\mathrm{a}}$ ), and nine different "dead-zone" populations, with varying radial extents $r_{\mathrm{dz}}$ and turbulence values $\alpha_{\mathrm{dz}}$. In Table 1, we list the parameter space explored for $r_{\mathrm{dz}}$ and $\alpha_{\mathrm{dz}}$. The active turbulence value of $\alpha_{\mathrm{a}}=10^{-3}$ was kept constant for all the simulations.

To construct the disk populations, we used a similar approach to the previous studies from Owen et al. (2011), Ercolano et al. (2018), and Picogna et al. (2019). Each population consists of 1000 simulations, where the X-ray luminosity is sampled from the Taurus luminosity distribution, with values between $L_{x}=$ $10^{28} \mathrm{erg} \mathrm{s}^{-1}$ and $L_{x}=10^{31} \mathrm{erg} \mathrm{s}^{-1}$ (Preibisch et al. 2005; Güdel et al. 2007), and the disk characteristic size was sampled from a uniform distribution with values between $r_{\mathrm{c}}=20 \mathrm{AU}$ and $r_{\mathrm{c}}=100 \mathrm{AU}$. To ensure that the populations are comparable with each other, we used the same sample of $L_{x}$ and $r_{\mathrm{c}}$ across the different populations.

We tracked the evolution of each simulation for $10 \mathrm{Myr}$, or until the photoevaporation carved a gap extending beyond $100 \mathrm{AU}$, saving a snapshot every $0.1 \mathrm{Myr}^{2}$. In particular, we wanted to obtain the probability distribution of the gas accretion rate $\dot{M}_{\mathrm{g}}$ (measured at $1 \mathrm{AU}$ ) and the outer edge of the gap opened by photoevaporation, including the presence of a dead zone, for each population model (see Sect. 4), and we compared them to the observed distribution of accreting transition disks.

\footnotetext{
2 The simulation data from the population synthesis and plotting rou-
} tines are available in Zenodo: doi .org/10.5281/zenodo. 4761432

\subsection{Model with dust evolution}

We performed an additional simulation including dust evolution (as described in Sect. 2.3) and using the following parameters: $L_{x}=10^{31} \mathrm{erg} \mathrm{s}^{-1}, r_{\mathrm{c}}=60 \mathrm{AU}, \alpha_{\mathrm{dz}}=10^{-4}$, and $r_{\mathrm{dz}}=5 \mathrm{AU}$. The remaining parameters are the same as in Sect. 3.1.

For the size distribution, we considered a logarithmic grid of $n_{m}=141$ particle species going from $5 \times 10^{-5} \mathrm{~cm}$ to $2.5 \times 10^{2} \mathrm{~cm}$. The initial dust size distribution follows the Mathis et al. (1977) power law, with a maximum size of $a_{0}=1 \mu \mathrm{m}$, and we assumed an initial dust-to-gas ratio of $\epsilon_{0}=0.01$.

For the dust properties, we assumed a fragmentation velocity of $v_{\text {frag }}=10 \mathrm{~m} \mathrm{~s}^{-1}$ (Wada et al. 2011; Gundlach et al. 2011; Gundlach \& Blum 2015), that the grains have a material density of $\rho_{\mathrm{s}}=1.6 \mathrm{~g} \mathrm{~cm}^{-3}$, and that these are compact, with the grain mass given by $m=4 \pi \rho_{\mathrm{s}} a^{3} / 3$. The dust then evolves according to the growth and advection model described in Sect. 2.3. Finally, we can use the dust size distribution to obtain the disk's SED and its brightness in both the millimeter continuum $(1.3 \mathrm{~mm})$ and scattered light $(1.25 \mu \mathrm{m})$, using the radiative transfer code RADMC- $3 D^{3}$ (Dullemond et al. 2012).

We restricted our analysis to a single "dust-and-gas" simulation for two reasons. Firstly, these simulations are computationally expensive to run. The second reason is that we are particularly interested in the scenario where a large gap opens on short timescales $(\approx 1 \mathrm{Myr})$, while keeping the presence of a compact inner dust disk, as this has been seen in observations of transition disks at different wavelengths (e.g., Benisty et al. 2010; Olofsson et al. 2013; GRAVITY Collaboration 2019, 2020; Pinilla et al. 2019; Francis \& van der Marel 2020; Rosotti et al. 2020). In order to ensure the fast gap opening, we used a high value for the X-ray luminosity $\left(L_{x}=10^{31} \mathrm{erg} \mathrm{s}^{-1}\right)$ in this simulation. We defer an in-depth study of the dust evolution to a follow-up work and limit ourselves to commenting on the expected effect of the existing parameters based on the available results.

\section{Results: gas evolution}

In this section, we present the results of the population synthesis study with gas-only simulations, showing that disks with dead zones are more likely to continue accreting during photoevaporative dispersal than those without. As a simplification, we refer to a disk as "accreting" if the accretion rate at $1 \mathrm{AU}$ is $\dot{M}_{\mathrm{g}} \geq 10^{-11} M_{\odot} \mathrm{yr}^{-1}$, and "non-accreting" otherwise. We chose this limit in order to be consistent with previous works (e.g., Owen et al. 2011) and observational detection limits (Alcalá et al. 2017).

\subsection{Proof of concept}

To first illustrate how dead zones allow for a dispersing disk to sustain a high accretion rate, we present the gas surface-density evolution of two disks (with and without a dead zone) in Fig. 3. Both disks were constructed following Sect. 3.1, with $r_{\mathrm{c}}=60 \mathrm{AU}$ and $L_{x}=10^{30} \mathrm{erg} \mathrm{s}^{-1}$. For the disk with a dead zone, we used an extent of $r_{\mathrm{dz}}=10 \mathrm{AU}$ and turbulence of $\alpha_{\mathrm{dz}}=10^{-4}$ (see Fig. 2).

Figure 3 shows how the inner and outer parts of the disk with a dead zone evolve on different timescales. The outer disk is dispersed once the local accretion rate drops below the photoevaporation mass-loss rate (Clarke et al. 2001), while the inner

\footnotetext{
3 www.ita.uni-heidelberg.de/ dullemond/software/ radmc-3d/
} 

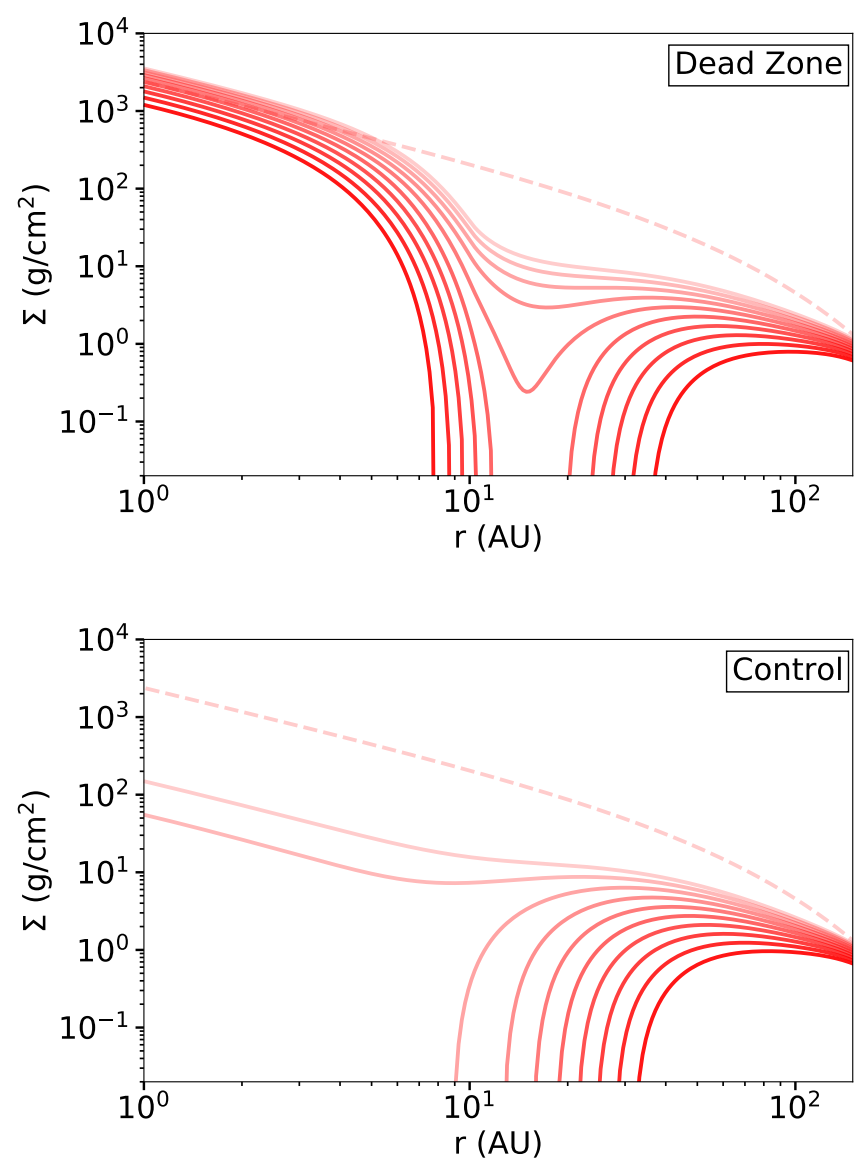

Fig. 3. Gas surface-density evolution of two simulations, with (top) and without (bottom) a dead zone, undergoing photoevaporative dispersal (with $L_{x}=10^{30} \mathrm{erg} \mathrm{s}^{-1}$ ). The dashed line shows the initial gas surface density. The solid lines show the gas evolution every $0.2 \mathrm{Myr}$, starting at $2.5 \mathrm{Myr}$, where the opacity increases with time. The dead-zone parameters for the top panel are $\alpha_{\mathrm{dz}}=10^{-4}$ and $r_{\mathrm{dz}}=10 \mathrm{AU}$.

disk evolves on a longer viscous timescale (Morishima 2012). In contrast, the inner region of the disk without a dead zone is accreted onto the star immediately after the gap opens.

Figure 4 shows how the disk with a dead zone maintains an accretion rate of $\dot{M}_{\mathrm{g}} \approx 10^{-9} M_{\odot} \mathrm{yr}^{-1}$ during its entire evolution, even after the inner disk is disconnected from the outer region. On the other hand, for the model without a dead zone the accretion rate drops quickly after the gap opens, turning it into a non-accreting disk.

\subsection{Population synthesis models}

The 2D histograms in Fig. 5 show the probability distribution of the accretion rate and gap size for two different population models of transition disks (each generated from 1000 simulations and sampling every $0.1 \mathrm{Myr}$ from the moment a gap opens, see Sect. 3.2). The control population, which consists of disks without a dead zone, shows that only a small fraction $(<1 \%)$ of the recorded transition disks are still accreting once the gap opens (with $\dot{M}_{\mathrm{g}}>10^{-11} M_{\odot} \mathrm{yr}^{-1}$ ). Also, once the gap exceeds $20 \mathrm{AU}$ in size, all transition disks have stopped accreting. The previous population studies of Owen et al. (2011) and Picogna et al. (2019) also show similar results.

The dead-zone population, on the other hand, shows that a high fraction of transition disks are accreting, with $31 \%$ of
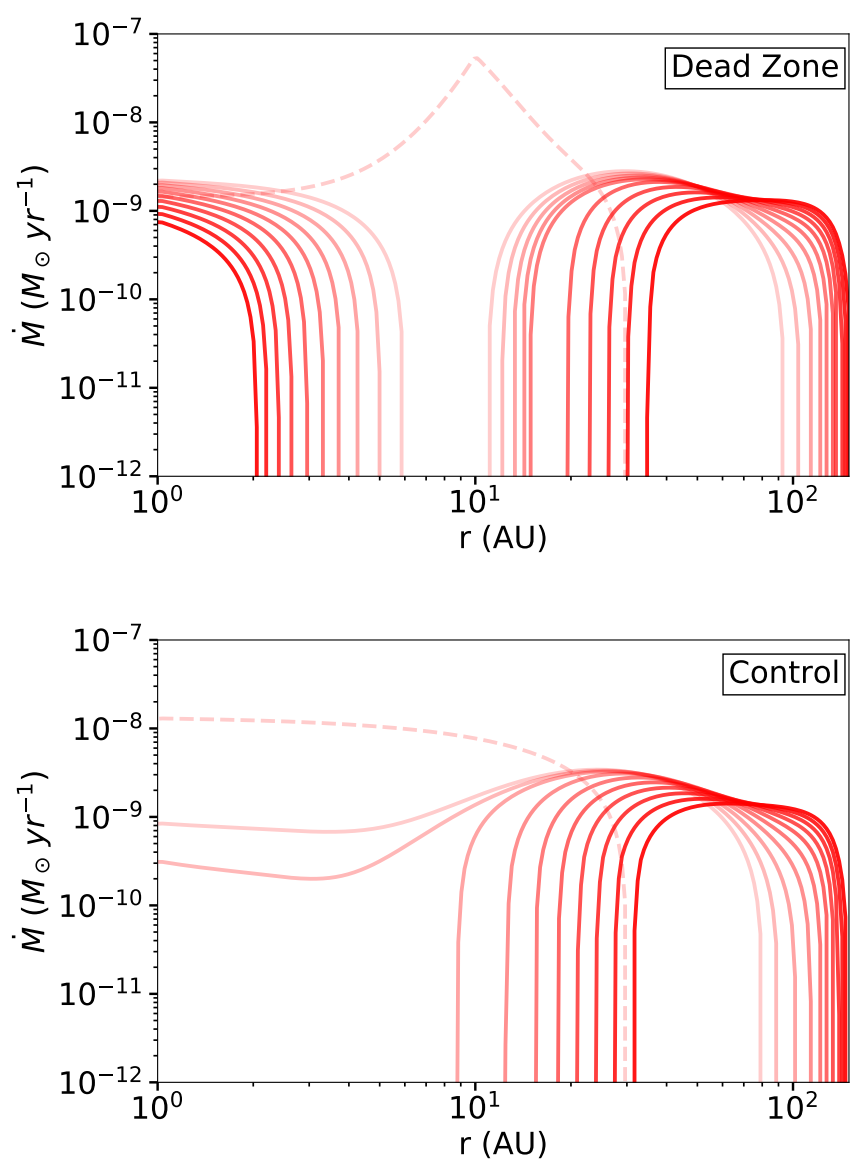

Fig. 4. Accretion-rate profiles for two simulations with (top) and without (bottom) a dead zone. The dashed line shows the initial accretion profile. The solid lines show the gas evolution every $0.2 \mathrm{Myr}$, starting at 2.5 Myr, where the opacity increases with time.

them accreting at rates of $\dot{M}_{\mathrm{g}}>5.0 \times 10^{-10} M_{\odot} \mathrm{yr}^{-1}$ and $63 \%$ at rates of $\dot{M}_{\mathrm{g}}>10^{-11} M_{\odot} \mathrm{yr}^{-1}$. We also find that the fraction of accreting transition disks is higher in disks with smaller gaps. In particular, if we only look at transition disks with gap sizes smaller than $50 \mathrm{AU}$, we find that $85 \%$ of them are accreting. In Fig. 5, we also over-plot the measured gap sizes and accretion rates of observed transition disk population (van der Marel et al. 2016b; Keane et al. 2014; Manara et al. 2014, 2016a,b, 2017; Garcia Lopez et al. 2006; Pascucci et al. 2007; Najita et al. 2007; Spezzi et al. 2008; Merín et al. 2010; Donehew \& Brittain 2011; Curran et al. 2011; Alcalá et al. 2014; Cieza et al. 2010, 2012; Romero et al. 2012; Follette et al. 2015) using the objects listed in Ercolano \& Pascucci (2017, Table A.1).

Comparing our results with the observed transition disks, we find that the model including a dead zone can reproduce the measured accretion rates and gap sizes larger than 10 AU. However, we also notice that this model does not predict disks with gaps smaller than the dead zone radial extent $r_{\mathrm{dz}}$ since the inner regions evolve too slowly for photoevaporation to carve a gap during the early stages of disk dispersal. In Sect. 6.1 we discuss how disks with narrow gaps could still be predicted within our framework.

In Fig. 6, we show the fraction of transition disks relative to the whole disk population (i.e. transition and primordial disks) at each time. In both the control and dead-zone populations, we find that photoevaporation opens a gap in approximately $20 \%$ of 


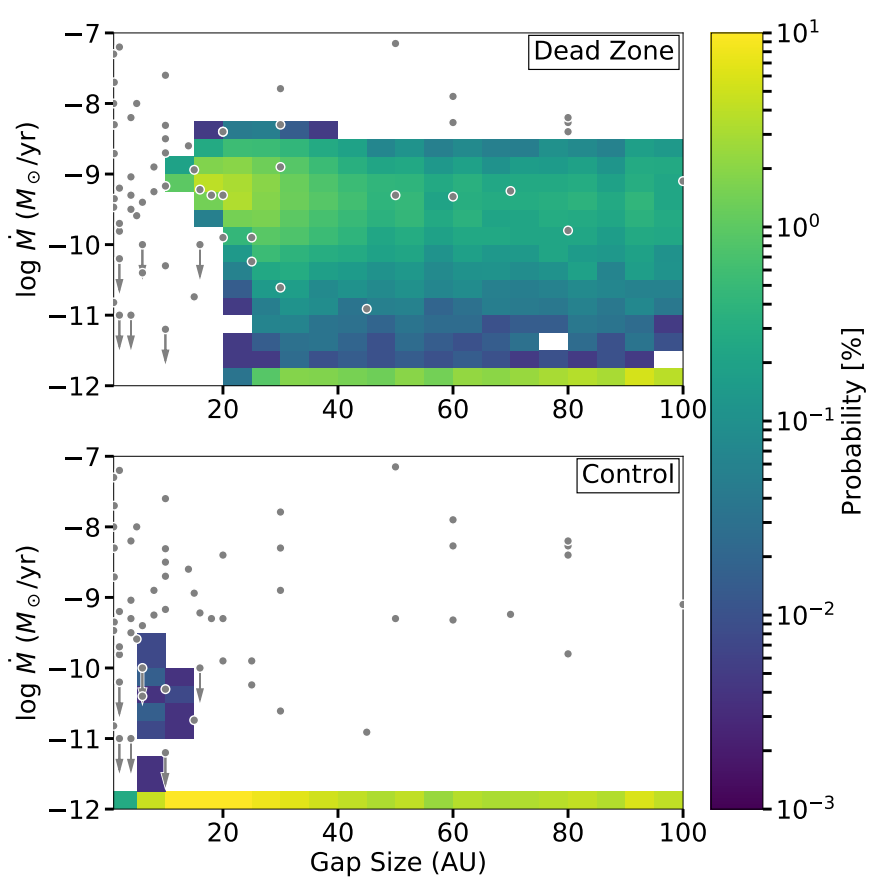

Fig. 5. Probability distribution of the gap size (measured with its outer edge) and accretion rate (measured at 1.5 AU) for a population synthesis model with (top) and without (bottom) a dead zone. The dead zone model uses $r_{\mathrm{dz}}=10 \mathrm{AU}$ and $\alpha_{\mathrm{dz}}=10^{-4}$. Disks with accretion rates lower than $10^{-12} M_{\odot} \mathrm{yr}^{-1}$ are counted in the lowest accretion-rate bin. The gray dots mark the observed population of transition disks compiled in the Ercolano \& Pascucci (2017) review. The dots with arrows mark upper limits in the accretion rate.

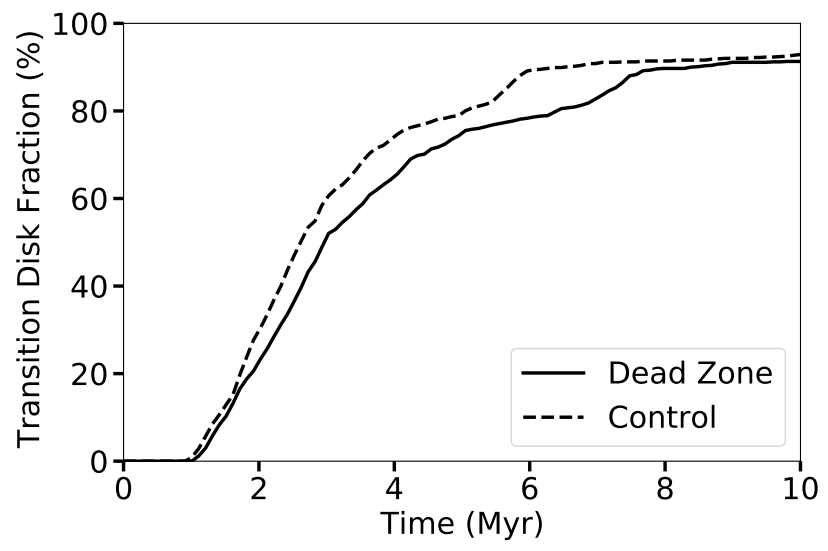

Fig. 6. Evolution of the fraction of transition disks over time, relative to the whole disk population, for both the control and dead-zone populations shown in Fig. 5. For simplicity, we identify a "transition disk" as a disk with a gap opened by photoevaporation.

the disks by $2 \mathrm{Myr}$, and in $90 \%$ of the disks at some point of their lifetime (i.e. within $10 \mathrm{Myr}$ ), indicating that while the dead zone helps to sustain the inner disk for longer timescales, it does not affect whether or not a gap opens, and this would be solely determined by the star's X-ray luminosity and the disk global properties (e.g., mass, size, and turbulence).

\subsection{Effect of the dead-zone properties}

To study how the properties of the dead zone affect the population of transition disks, we conduct a parameter space study for different dead zones sizes $\left(r_{\mathrm{dz}}\right)$ and turbulence parameters $\left(\alpha_{\mathrm{dz}}\right)$, shown in Fig. 7. To complement the figure, in Table 2 we include the fraction of transition disks that present accretion rates higher than $10^{-11} M_{\odot} \mathrm{yr}^{-1}$ for the different dead-zone properties. In all cases, we see a higher fraction of accreting transition disks when a dead zone is considered. Even the compact dead zone population with high turbulence $\left(\alpha_{\mathrm{dz}}=5 \times 10^{-4}\right.$ and $r_{\mathrm{dz}}=5 \mathrm{AU}$ ) shows $\approx 20$ times more accreting disks than the control population without a dead zone.

From the distribution of accretion rates and gap sizes, we learn that disks with low turbulence $\left(\alpha_{\mathrm{dz}}=10^{-4}\right)$ present the higher fraction of accreting transition disks, which is expected since the inner disk will evolve on longer viscous timescales. The effect of the dead-zone size is less straightforward, with mediumsized dead zones $\left(r_{\mathrm{dz}}=10 \mathrm{AU}\right)$ producing the highest fraction of accreting transition disks for a fixed $\alpha_{\mathrm{dz}}$ (see Table 2). Intuitively, we would expect larger dead zones to have longer viscous evolution timescales, with $t_{v} \sim r_{\mathrm{dz}}^{2} / v\left(r_{\mathrm{dz}}\right)$, and therefore to survive for longer, which is indeed what happens when we increase the dead-zone size from 5 to $10 \mathrm{AU}$. However, when further increasing the dead-zone size from 10 to $20 \mathrm{AU}$, we find that the fraction of accreting disks decreases from 63 to $55 \%$ (for $\alpha_{\mathrm{dz}}=10^{-4}$ ). We can explain this behavior if we consider that before photoevaporation opens a gap, dead zones evolve together with the whole disk, receiving material from the outer regions which is then redistributed across the inner regions within the local viscous timescale. In mid-sized dead zones the material is redistributed more efficiently than in more extended ones, resulting in a higher and spatially uniform accretion rate across the inner disk, and a longer lifetime. In contrast, for more extended dead zones the incoming material from the outer disk stays concentrated close around the dead zone's outer edge $\left(r_{\mathrm{dz}}\right)$ for timescales of several million years. The innermost region then evolves isolated from the outer disk, allowing photoevaporation to open a gap within the dead zone itself, and reducing the fraction of accreting transition disks. Additionally, we also find that the fraction of accreting transition disks can be higher if the initial mass distribution follows a quasi-steady state solution (see Appendix A).

\section{Results: dust evolution}

In this section, we describe the evolution of the dust component for a disk with a dead zone (following the setup from Sect. 3.3) and show the expected emission using radiative transfer calculations.

\subsection{Dust distribution}

Figure 8 shows how the dust surface density (integrated for all the grain sizes) evolves along with the gas during the disk dispersal in the case of a strong X-ray luminosity $\left(L_{x}=10^{31} \mathrm{erg} \mathrm{s}^{-1}\right)$. Before the gap opening, the dust drifts from the outer disk toward the inner-dead-zone regions, as it loses angular momentum due to the drag force from the gas (Whipple 1972; Weidenschilling 1977). Because of the low turbulence in the dead zone region, dust particles can grow to larger sizes and therefore drift faster, depleting the inner disk of solids on shorter timescales (Eqs. (6) and (11)). The dust size distribution (Fig. 9, top panel) shows that in the dead zone the dust particles can grow up to sizes of $a \sim 10 \mathrm{~cm}$, while in the outer disk we find mostly millimeter and submillimeter grains.

Additionally, we notice that our dead-zone model results in a disk where particle growth is completely limited by drift, whereas disks without dead zones would be typically dominated 

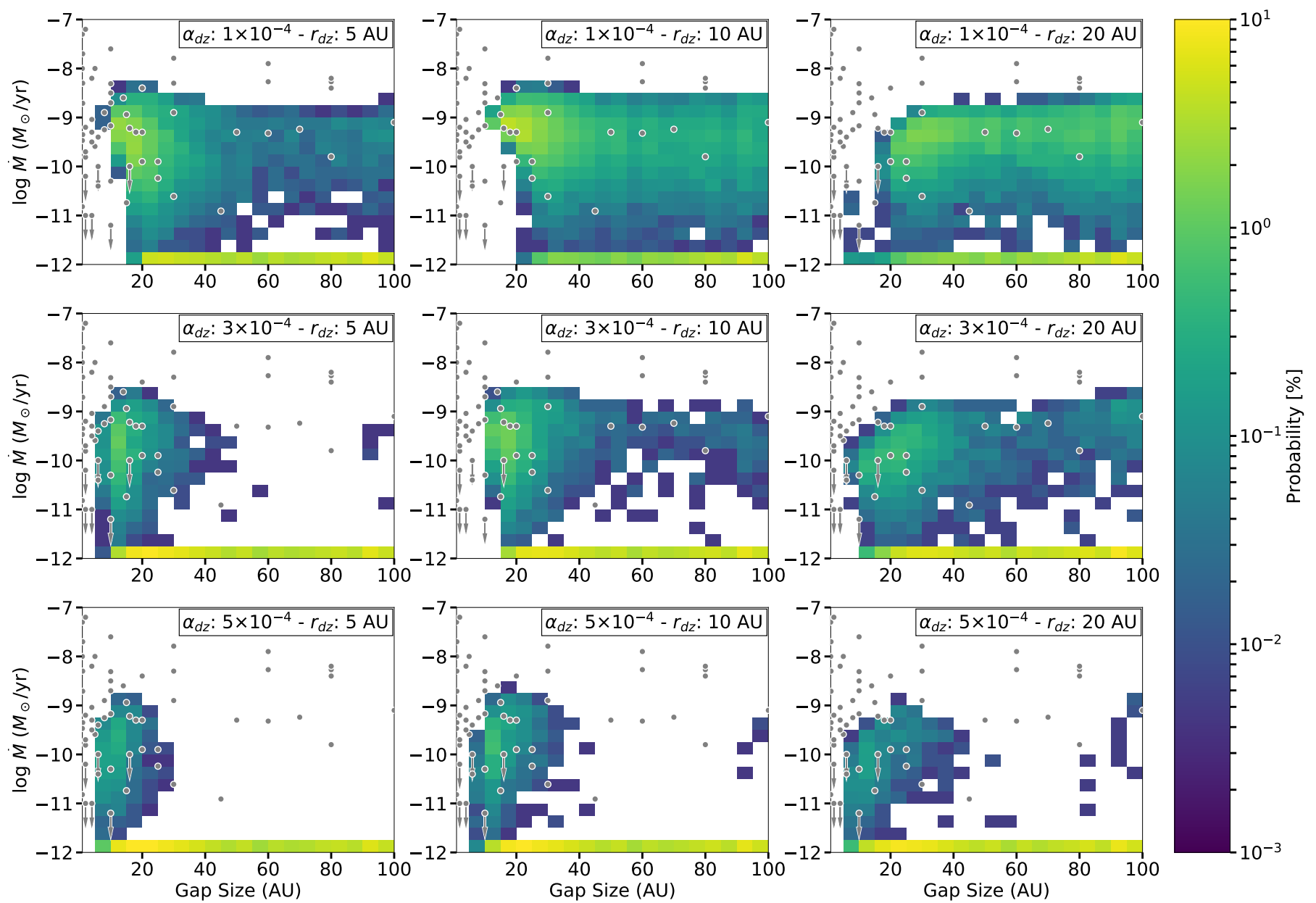

Fig. 7. Same as Fig. 5, but for different dead-zone models. The labels indicate the $\alpha_{\mathrm{dz}}$ and $r_{\mathrm{dz}}$ parameters used in each model.

Table 2. Percentage of transition disks with $\dot{M}_{\mathrm{g}}>10^{-11} M_{\odot} \mathrm{yr}^{-1}$ for different dead zone models.

\begin{tabular}{ccc}
\hline \hline$\alpha_{\mathrm{dz}}$ & $r_{\mathrm{dz}}(\mathrm{AU})$ & Accreting disk fraction $(\%)$ \\
\hline \multirow{3}{*}{$10^{-4}$} & 20 & 55.2 \\
& 10 & 62.9 \\
& 5 & 34.1 \\
\hline \multirow{3}{*}{$3 \times 10^{-4}$} & 20 & 12.3 \\
& 10 & 16.9 \\
& 5 & 8.9 \\
\hline \multirow{3}{*}{$5 \times 10^{-4}$} & 20 & 2.4 \\
& 10 & 4.6 \\
\hline Control & 5 & 3.0 \\
\hline
\end{tabular}

by drift only in the outer regions $\left(r \gtrsim 10\right.$ AU for $\alpha=10^{-3}$, after $1 \mathrm{Myr}$ ), and by fragmentation in the inner ones (Birnstiel et al. 2010, 2012b).

Once the photoevaporation opens a gap (approximately at $1 \mathrm{Myr}$ ), the inner and outer disk become disconnected in terms of mass transfer. The inner disk continues to lose material as the dust drifts inward, though we also observe that the drift timescales increase over time. This happens because when the dust-to-gas ratio decreases, the maximum Stokes number

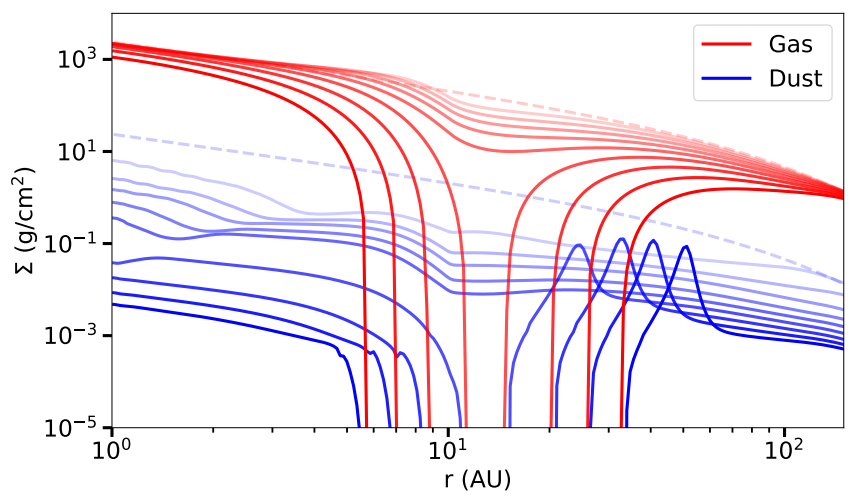

Fig. 8. Surface-density evolution for gas and dust (integrated for all particle sizes) in a disk with a dead zone (as described in Sect. 3.3). The dashed line shows the initial condition, the solid lines show the disk evolution every $0.2 \mathrm{Myr}$. The star has an X-ray luminosity of $L_{x}=$ $10^{31} \mathrm{erg} \mathrm{s}^{-1}$, which leads to a gap opening at $t \approx 1 \mathrm{Myr}$.

decreases as well (Eq. (11)), which leads to slower drift velocities (Eq. (6)). This behavior is in line with the arguments described in Birnstiel et al. (2012b) and Powell et al. (2017), in which the lifetime of disks with grain growth limited by drift is approximately equal to the growth and drift timescales. Since the growth timescale is approximately $t_{\text {growth }}=\left(\epsilon \Omega_{\mathrm{k}}\right)^{-1}$, we find that the time elapsed after the gap opening (i.e., the lifetime of the 

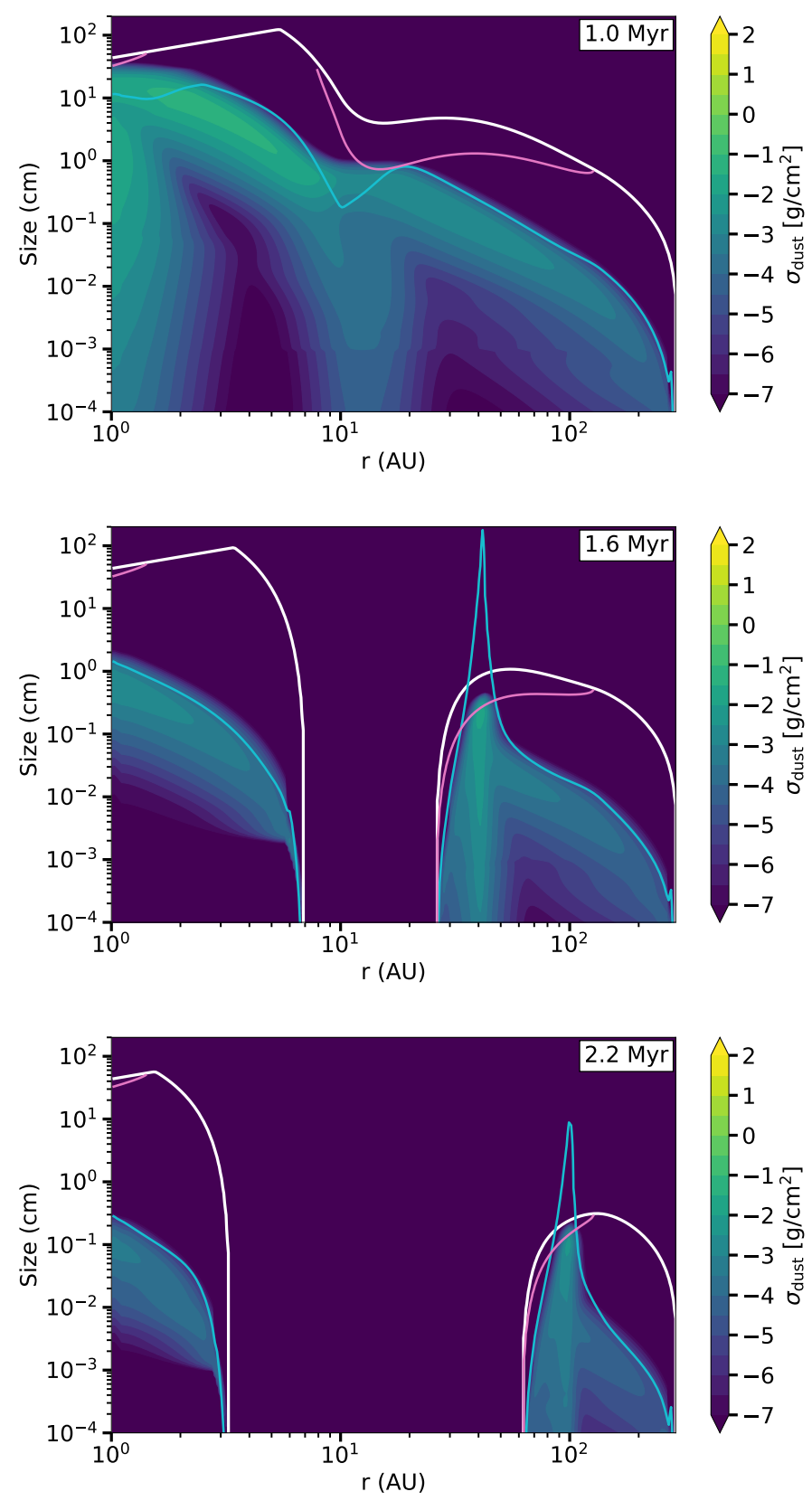

Fig. 9. Dust size distribution for a disk with a dead zone (as described in Sect. 3.3) at 1.0, 1.6, and 2.2 Myr. The solid lines show the drift growth limit (cyan), the fragmentation growth limit (pink), and the size corresponding to $\mathrm{St}=1$ (white).

inner disk as an isolated system) determines the dust-to-gas ratio present in the inner regions.

From the dust distribution of the inner region (Fig. 9, middle and bottom panels), we observe that it is dominated mostly by large centimeter-sized grains, and that there is a lack of micronsized dust. This happens because the collision velocities in the dead zone are low (Eq. (13)) and prevent the small particles being replenished by fragmentation.

Meanwhile, the solids in the outer disk drift toward the edge of the photoevaporative gap, which is the new local pressure maximum. The dust grains form a ring in which growth is limited by fragmentation, reaching sizes of $a \approx 1 \mathrm{~mm}$. As the gap expands over time, the dust ring moves along with it (Alexander \& Armitage 2007; Owen \& Kollmeier 2019).
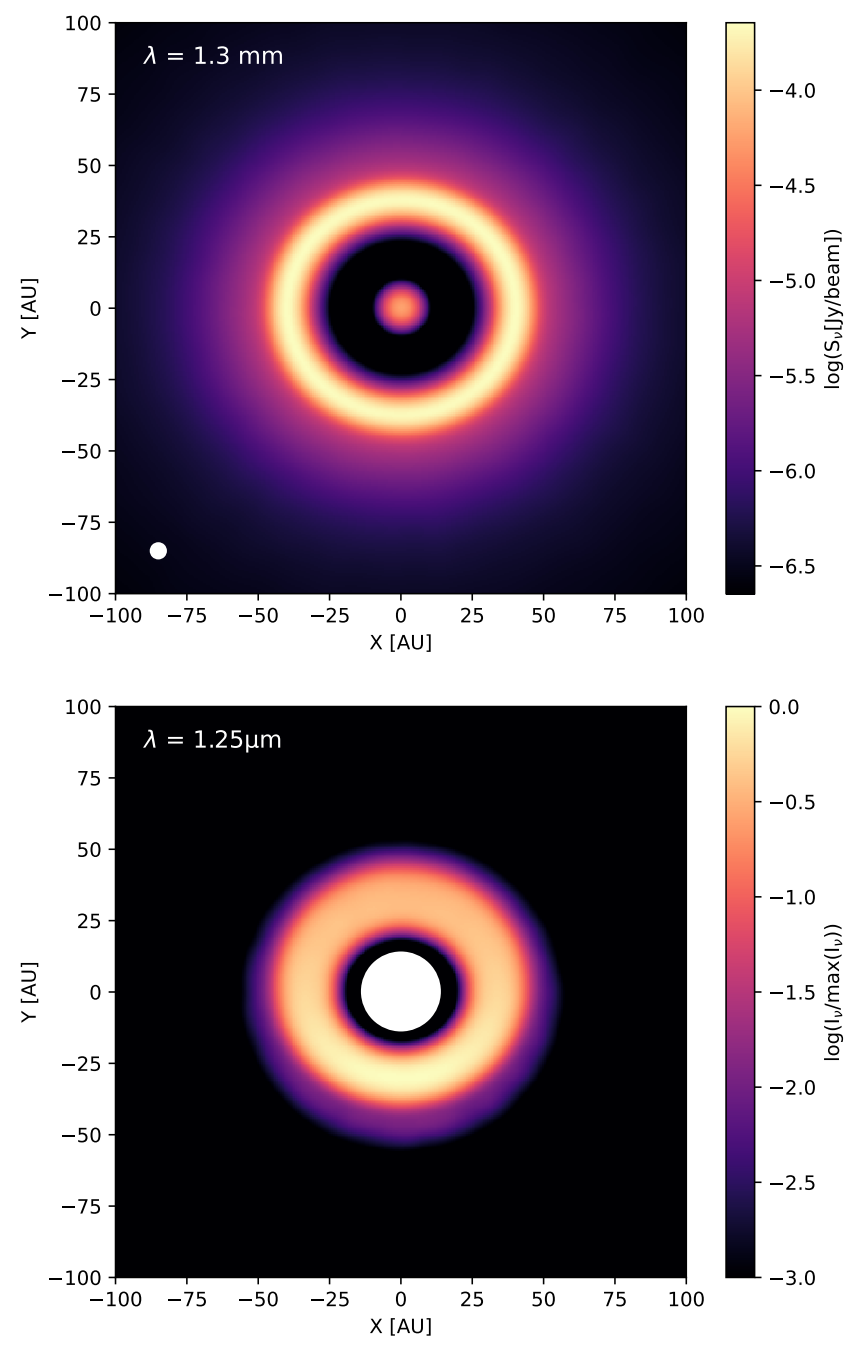

Fig. 10. Synthetic images of the radiative transfer models for the dust size distribution presented in Sect. 5.1 at $t=1.6 \mathrm{Myr}$, both convolved with a Gaussian beam of 40 mas. Top: continuum emission at $\lambda=1.3 \mathrm{~mm}$; the beam size is shown in the lower left corner. Bottom: scattered light brightness at $\lambda=1.25 \mu \mathrm{m}$, normalized to the peak emission of the ring. The inner disk is covered by a $0.1^{\prime \prime}$ coronograph.

\subsection{Dust emission}

Using the dust size distributions obtained with DustPy in Sect. 5.1, we performed radiative transfer calculations using the code RADMC-3D. We calculated the opacities for each grain size using the program $0 \mathrm{pTool}^{4}$, assuming compact spheres and a dust composition consisting of water ice (Warren \& Brandt 2008), astronomical silicates (Draine 2006), troilite, and refractory organics (Henning \& Stognienko 1996), as stated in Birnstiel et al. (2018). To account for the problems caused by the strong forward-scattering peak in the phase function of large grains and the limited spatial resolution or our model, we set the scattering opacity to an upper limit within the first $5^{\circ}$ of the phase function. We assumed the solar type star to be a blackbody point source and used $10^{7}$ photons for our radiative transfer calculations. First, the 3D dust temperature structure was computed and then used to calculate the synthetic images and SEDs with the full treatment of anisotropic scattering with polarization (Kataoka et al. 2015), as shown in Figs. 10 and 11,

4 github.com/cdominik/optool, version 1.7.2 


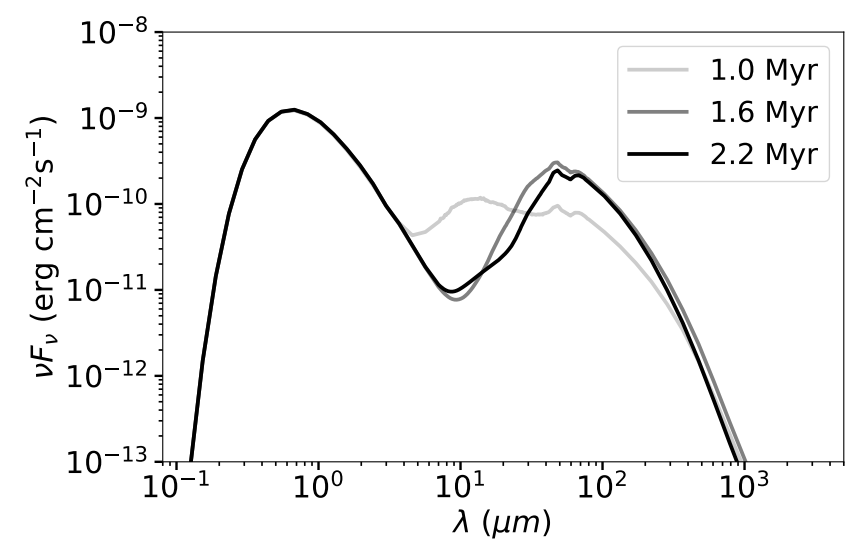

Fig. 11. Spectral energy distribution for the disk model described in Sect. 5.1 at three different times. The disk is assumed to be at a distance of $140 \mathrm{pc}$ and at an inclination of $20^{\circ}$.

respectively. The disk is assumed to have an inclination of $20^{\circ}$ and is observed at a distance of $140 \mathrm{pc}$, which is the mean value of the nearest star forming regions. Finally, both images were convolved using a Gaussian point-spread function with a full width at half maximum $(\mathrm{FWHM})$ of $0.04^{\prime \prime} \times 0.04^{\prime \prime}$ to mimic the current angular resolution obtained by instruments such as the Spectro-Polarimetric High-contrast Exoplanet Research (SPHERE) at the Very Large Telescope (VLT) and the Atama Large Millimeter/submillimeter Array (ALMA).

Figure 10 (top panel) shows the $1.3 \mathrm{~mm}$ continuum emission of our model after the gap opening (at $1.6 \mathrm{Myr}$ ). We observe a signal coming from the inner disk $(r \approx 10 \mathrm{AU})$ where the dead zone is located and from a ring of dust located at $r \approx 40 \mathrm{AU}$, which corresponds to the pressure trap at the outer edge of the photoevaporative gap. The region in between is devoid of any emission, as is expected from the dust distribution seen in Fig. 9.

The total emission in the $1.3 \mathrm{~mm}$ continuum disk is $F_{\mathrm{mm}}=$ $14.2 \mathrm{mJy}$. In terms of the classification of transition disks according to their fluxes (Owen et al. 2012), our model would be classified as a millimeter-faint disk $\left(F_{\mathrm{mm}} \leq 30 \mathrm{mJy}\right)$. This is at odds with the observed properties of disks with large gaps $\left(r_{\text {gap }} \gtrsim 20 \mathrm{AU}\right)$ and high accretion rates $\left(\dot{M}_{\mathrm{g}} \sim 10^{-9} M_{\odot} \mathrm{yr}^{-1}\right)$, which are more likely to have millimeter fluxes above $30 \mathrm{mJy}$ (see Owen 2016, Fig. 5). We discuss the implications of the low millimeter flux found in our model in Sect. 6.1.

The scattered light image (Fig. 10, bottom panel) shows a wide ring that extends approximately between 25 and $50 \mathrm{AU}$. There is a signal coming from the inner disk in the scattered light, which is mainly occluded by the chronograph that we assume (0.1" in size, which is typical for observations with SPHERE).

Figure 11 shows the evolution of the SED. The SED presents a decrease in the IR emission between 1.0 and $1.6 \mathrm{Myr}$, approximately between $5 \mu \mathrm{m}$ and $30 \mu \mathrm{m}$, which coincides with the gap opening and the depletion of micron-sized particles in the inner disk (see Fig. 9). This feature is characteristic of transition disks, and this object would be classified as such from its SED (Strom et al. 1989; Espaillat et al. 2014).

\section{Discussion}

\subsection{Comparison with the observed transition disk population}

The discrepancy between the predictions of theoretical models and the observed population of transition disks has been an open problem for the last decade. Population synthesis models including photoevaporation predict that $88-97 \%$ of transition disks should be non-accreting disks with large gaps (Owen et al. 2011; Picogna et al. 2019), also called "relic disks". Observational constraints, on the other hand, say that the fraction of these nonaccreting relics should only be around 3\% (Hardy et al. 2015). In the literature, this discrepancy can also be found as the "relic disk problem".

Recent works from Ercolano \& Clarke (2010), Ercolano et al. (2018), and Wölfer et al. (2019) suggest that photoevaporation is more effective in disks that are depleted from carbon and oxygen, which results in higher mass-loss rates and a faster dispersal of the outer disk. The outcome of this approach is a lower fraction of relics (up to $45 \%$ ), which is much lower than standard photoevaporative models, and better suited to explain observations.

Meanwhile, models including dead zones and grain growth have also been able to reproduce some of the features observed in transition disks, including the decrease in the NIR emission due to inefficient fragmentation, and ring like structures (Birnstiel et al. 2012a; Pinilla et al. 2016a); however, these models also fail to clear extended gaps in the millimeter continuum, because the drift timescales of the large grains become too long, or because the particle trapping occurs beyond the dead zone's outer edge.

In this work we present a different approach, which consists of combining the photoevaporative dispersal by X-ray radiation, with a dead zone in the inner disk. This way, both processes complement each other by covering their respective weak spots: the dead zone takes care of extending the lifetime of the inner disk, keeping the accretion rates high, while photoevaporation carves extended gaps in the gas and dust components (see also Morishima 2012).

The strongest point of this model is that it produces a high fraction of disks with large gaps $\left(r_{\text {gap }} \gtrsim 20 \mathrm{AU}\right)$ and high accretion rates $\left(\dot{M}_{\mathrm{g}} \sim 10^{-9} M_{\odot} \mathrm{yr}^{-1}\right)$ at the same time, predicting that up to $63 \%$ of all transition disks should be accreting. This fraction is higher than previous population synthesis estimates, though it is still not high enough to match the observational constrains by itself (Hardy et al. 2015) since we still find that $37 \%$ of transition disks should be relics (i.e., non-accreting).

The predicted fraction of relic disks could be even lower if the effects of carbon depletion are considered, with the respective increase in the mass-loss rate and faster dispersal of the outer disk (Ercolano et al. 2018), or if the disks inner regions are able to survive for longer times, by reaching a quasi-steady state during earlier stages of disk evolution, for example (see Appendix A).

Alternatively, it could also be that many disks become too faint to be observed if dust is removed efficiently, either by drift during early stages of disk evolution, or by a strong coupling with the photoevaporative wind (Owen \& Kollmeier 2019). While in our simulations the dust-loss rate is always too low to affect the total dust mass, a more detailed modeling of the gas drag at the edge of the photoevaporative gap could yield higher dustloss rates at lower scale heights, after the dispersal of the inner disk.

In the context of the whole disk population (i.e., counting primordial and transition disks), our results seem to match the fraction of transition disks shown in Currie \& Sicilia-Aguilar (2011), especially around 2 Myrs, with the dead zone having little to no influence on whether a gap is opened or not. At later times, we do predict more transition disks than those observed, but this discrepancy could also be bridged if we consider that some of our disk should become fainter over time, again due to dust evolution, entrainment, and drifting. 
We should keep in mind that the accuracy of our predictions depends, of course, on the validity of our dead-zone model (Eq. (4)), since the fraction of accreting transition disks varies greatly across different dead-zone parameters (see Table 2). We discuss this point in Sect. 6.3, where we calculate what a self-consistent dead-zone model would look like.

One apparent limitation of our model is that it is unable to produce enough transition disks with narrow gaps $\left(r_{\text {gap }} \lesssim 10 \mathrm{AU}\right.$, see Fig. 7), since by construction the combined effects of dead zones and photoevaporation favor opening a gap beyond the dead zone's outer edge (Morishima 2012). This seems at odds with the observations, which indicate there are approximately as many transition disks with gaps that are narrower than $10 \mathrm{AU}$ as disks with wider gaps and sizes between 10 and $100 \mathrm{AU}$ (van der Marel et al. 2016b). However, most of the gaps in transition disks with sizes smaller than $10 \mathrm{AU}$ are still unresolved by observations, and they are characterized by SED fitting. This means that the current gap sizes reflect the deficit of small dust grains in the inner regions but may not correspond to an actual gap in the gas or in the millimeter continuum. We could still predict this type of narrow gap within our framework, if we consider that dust growth in dead zones can deplete the inner regions of small grains and create SED signatures that are akin to that of a transition disk (Birnstiel et al. 2012a). Therefore, a disk could display a narrow gap signature in their SED due to a dead zone at earlier times, and a wide gap signature in both their SED and millimeter continuum at later stages due to the combined dead-zone and photoevaporation effects described in Morishima (2012) and this work.

Besides the accretion rate and gap sizes, the observed transition disks seem to be divided into two groups according to their millimeter flux $F_{\text {mm }}$ (Owen et al. 2012). Disks with smaller gaps tend to be "millimeter-faint" $\left(F_{\mathrm{mm}}<30 \mathrm{mJy}\right)$, while disks with larger cavities are "millimeter-bright" $\left(F_{\mathrm{mm}}>30 \mathrm{mJy}\right)$, with the fluxes rescaled to a distance of 140 pc (see Owen 2016, Figs. 3 and 5). Instead, the millimeter flux of our dust evolution model is only $F_{\mathrm{mm}}=14.2 \mathrm{mJy}$, despite it presenting a large cavity and a high accretion rate. Simulations with different parameters that were not included in this paper also show similar fluxes, around $10 \mathrm{mJy}$. This suggests that the dust content in our disks is relatively low in comparison with with the observed transition disks, or that we are underestimating the dust opacity.

We can explain why our disks appear to be dust depleted by looking at our initial gas density profile (Eq. (14)), which is smooth and does not have any substructure. In such a disk, the dust particles can drift inwards and into the star very quickly, since there are no pressure maxima to act as local dust traps (Pinilla et al. 2012b). In contrast, observations of protoplanetary disks show that these are rich in substructures such as rings and spirals (e.g., ALMA Partnership 2015; Andrews et al. 2018), indicating that the underlying gas profile is not smooth. Recent models also indicate that dust traps are necessary in order to explain the size luminosity relationship observed in protoplanetary disks (Tripathi et al. 2017; Zormpas et al., in prep.). Including pressure bumps in our model at early times, such as a gap created by a planet, would help our disk to retain a higher fraction of dust, increase its millimeter emission, and reduce the discrepancy with observations (Pinilla et al. 2012a, 2020).

Despite this limitation in our model, it seems that the presence of a dead zone in the inner regions is a promising ingredient to explain the observed transition disk population. For a follow-up work, we intend to quantify the millimeter continuum emission of disks undergoing photovaporative dispersal and test if it is possible to retain enough solids to match the observed fluxes from transition disks through trapping (Pinilla et al. 2020).

Additional improvements for the model would be to include stars of different masses, considering their respective photoevaporation mass-loss rates (Komaki et al. 2021), the evolution of the stellar luminosity during the first million years after formation (Kunitomo et al. 2021), and the effect of carbon depletion, which leads to stronger photoevaporation rates (Ercolano et al. 2018; Wölfer et al. 2019).

\subsection{Long-lived inner disk and comparison with observations}

The large diversity in the SED morphology of transition disks (e.g., Cieza et al. 2007) provides evidence that the cavity of some of these disks is not completely depleted in dust. Some of the SEDs of transition disks exhibit a strong excess in the NIR wavelength range (e.g., Espaillat et al. 2010; van der Marel et al. 2016b), implying a compact, optically thick inner disk very close to the star.

Observational efforts have been made to directly observe and characterize the inner disk of transition disks. For example, interferometric observations at the NIR have spatially resolved a compact inner disk in some transition disks (e.g., Benisty et al. 2010; Olofsson et al. 2013; GRAVITY Collaboration 2019, 2020). Recent observations with ALMA also detect, and in a few cases resolve, the inner disk of some transition disks, meaning that these inner disks are probably rich in millimeter-sized particles or that there is an equivalent amount of smaller grains that lead to a similar signal in the millimeter continuum (e.g., Hendler et al. 2018; Pinilla et al. 2019; Keppler et al. 2019; Francis \& van der Marel 2020; Rosotti et al. 2020).

The physical mechanism responsible for the observed dust in the inner disk of transition disks is an open question, and it is unclear how it can survive on timescales of millions of years. For the formation of the large gaps in these objects, three main possibilities are debated in the literature: embedded planets, photoevaporation, and dead zones. However, each of these scenarios have their own challenges to explain a long-lived inner disk as observed at different wavelengths.

On one hand, the planet-disk interaction models may allow us to have partial dust filtration at the outer edge of the gap (Pinilla et al. 2012a; Weber et al. 2018), such that the inner disk can be replenished with small dust (micron-sized) from the outer disk. However, in this scenario these small particles grow very efficiently in the inner disk once they cross the gap and thus they are lost toward the star due to efficient drift. This problem can be mitigated by including the effect of the snow line, which can change the velocities for which particles fragment (Wada et al. 2011; Gundlach et al. 2011; Gundlach \& Blum 2015) and hence slow down their radial drift (Birnstiel et al. 2010; Pinilla et al. 2016b; Drążkowska \& Alibert 2017). These models of planets with the snow line are capable of reproducing the NIR excess seen in the SEDs of some transition disks, but they fail in reproducing a detectable inner disk at millimeter wavelengths (see Fig. A.1 in Pinilla et al. 2016b).

On the other hand, models of dead zones acting alone can reproduce an inner dusty disk, but it is expected to be much fainter than the ring-like emission in the outer disk at millimeter wavelengths (see Figs. 7 and 8 in Pinilla et al. 2016a). Additionally, in these models there is not a clear depletion of the gas surface density inside the millimeter cavity, which seems to be highly depleted from observations of ${ }^{12} \mathrm{CO}$ and its isotopologues (van der Marel et al. 2016a). To solve this problem, it was suggested that the inclusion of an MHD wind in the simulations 
could reduce the gas surface density in the inner regions, but that it would worsen the problem of keeping a long-lived inner disk.

Finally, photoevaporation models prior to this work predicted highly depleted cavities, in both gas and dust (Alexander \& Armitage 2007; Owen \& Kollmeier 2019), but these ignored the combined effect of a potential dead zone in the photoevaporative dispersal process. The models presented in this paper can explain a dusty inner disk that remains for millions of years and that is bright at millimeter emission.

The maximum grain size in the inner disk is limited by drift, and therefore by the local dust-to-gas ratio, which is gradually decreasing with time. As a consequence, the radial drift velocity of the particles in the inner disk also decreases, prolonging their lifetime (Fig. 9). This solves the conundrum of the origin and survival of these inner disks in transition disks.

From our synthetic observations at millimeter wavelengths (Fig. 10), we find that the surface brightness in the inner disk is slightly fainter (by a factor of a few) than the ring at the outer edge of the photoevaporative gap. A similar feature has been observed in some transition disks (e.g., TCha and CIDA1, Hendler et al. 2018; Pinilla et al. 2021, respectively), where the inner disk has a comparable brightness to the outer disk. The millimeter-sized particles in the inner regions extend until the outer edge of the inner disk, as given by the gas distribution, indicating that the dust can be retained over long timescales (along with the gas component) without the need for additional dust traps.

\subsection{Validity of the dead-zone model}

In this work, we modeled the presence of a static dead zone using the $\alpha(r)$ profile described in Eq. (4), which is defined by the free parameters $\alpha_{\mathrm{dz}}, \alpha_{\mathrm{a}}$, and $r_{\mathrm{dz}}$. Our parameter space study in Sect. 4 shows that the fraction of accreting transition disks is heavily influenced by the turbulence in the dead zone, $\alpha_{\mathrm{dz}}$, and the dead zone extent, $r_{\mathrm{dz}}$. For this reason, we are interested to see if a more complex model could produce similar profiles in terms of the dead-zone turbulence and radial extent.

We performed a quick validation test using the framework developed in Delage et al. (in prep.). The authors built a 1+1D magnetically driven disk-accretion model for the outer disk regions ( $r \gtrsim 1 \mathrm{AU}$ ), where the local mass and angular momentum transport are assumed to be controlled solely by the MRI and hydrodynamic instabilities such as, for example, the VSI. From such a model, we can derive an effective turbulent parameter $\bar{\alpha}$ that is self-consistently determined from detailed considerations of the MRI and the nonideal MHD effects (Ohmic resistivity and Ambipolar diffusion), given stellar properties (mass and luminosity), disk mass, and dust properties. Their model accounts for the main physical processes happening in the outer regions of protoplanetary disks: (1) irradiation from the forming star; (2) dust settling; (3) ionization from stellar X-rays, galactic cosmic rays, and the decay of short- and long-lived radionuclides; and (4) disk chemistry. Using their framework, we investigated two scenarios. First, the effective turbulence $\bar{\alpha}$ was calculated from a gas surface density following the standard self-similar solution from Lynden-Bell \& Pringle (1974). Since this scenario leads to a disk structure that is out of equilibrium (the accretion rate is radially nonconstant, implying that some regions of the disk can accrete faster than others), we considered a second scenario where the effective turbulence $\bar{\alpha}$ is derived by assuming steady-state accretion. In the latter, the gas surface density is self-consistently derived through an iterative process, alongside

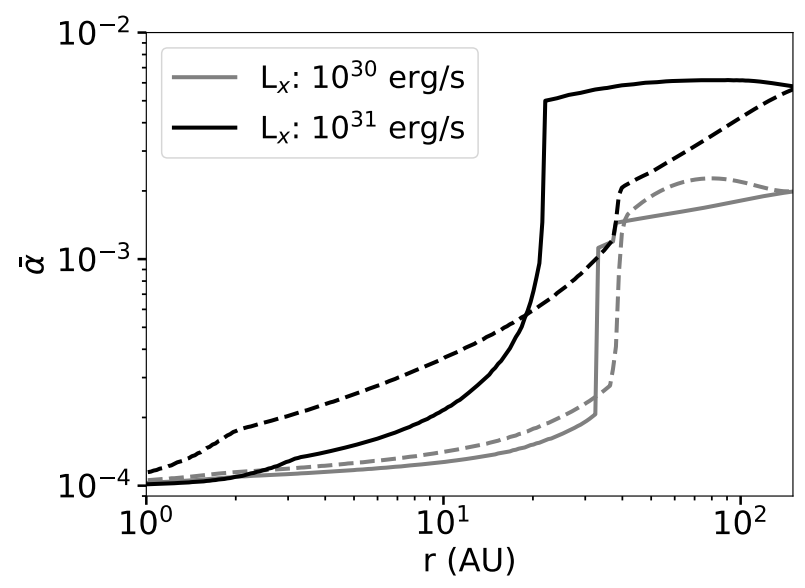

Fig. 12. Effective turbulence $\bar{\alpha}$ profile for different X-ray luminosities, derived using the MRI model described in Delage et al. (in prep.). The solid and dashed lines correspond to the steady-state scenario and the Lynden-Bell \& Pringle (1974) scenario, respectively. See Sect. 6.3.

the effective turbulence $\bar{\alpha}$, to ensure the accretion rate is radially constant. For each scenario, we considered two X-ray luminosities $\left(10^{30}\right.$ and $\left.10^{31} \mathrm{erg} \mathrm{s}^{-1}\right)$. The stellar and disk mass are the same as described in Sect. 3.1. We further assumed a constant dust-to-gas ratio of $\epsilon=0.01$, as well as grains of size $a=1 \mu \mathrm{m}$, consistently with the setup described in Sect. 3.3.

Figure 12 shows the $\alpha(r)$ profiles derived for the four simulations, using the framework described in Delage et al. (in prep.). Depending on the X-ray luminosity used or the scenario considered, the salient results are as follows: the dead zone extent $r_{\mathrm{dz}}$ ranges from 22 to $40 \mathrm{AU}$, where the turbulence quickly changes from a low to high regime; the mean turbulence in the dead zone $\alpha_{\mathrm{dz}}$ ranges from $1.3 \times 10^{-4}$ to $3.7 \times 10^{-4}$; and the mean turbulence in the MRI-layer $\alpha_{\mathrm{a}}$ ranges from $1.8 \times 10^{-3}$ to $5.7 \times 10^{-3}$.

Based on these calculations, we find that the dead-zone radial extent should be larger than the one used in our models, which in principle would mean that the fraction of accreting transition disks could be better represented by populations with $r_{\mathrm{dz}}=20 \mathrm{AU}$. We also find that the mean values for the turbulence in the dead zone are covered by our parameter space exploration. Furthermore, the highest value found $\left(\alpha_{\mathrm{dz}}=3.7 \times 10^{-4}\right)$ would suggest that our choice of $\alpha_{\mathrm{dz}}=5 \times 10^{-4}$ is quite marginal, and for moderate X-ray luminosities $\left(L_{x}=10^{30} \mathrm{erg} \mathrm{s}^{-1}\right)$ the deadzone turbulence remains between $\alpha_{\mathrm{dz}}=10^{-4}$ and $2 \times 10^{-4}$. We expect that a population synthesis model using the dead-zone model from Delage et al. (in prep.) would also present gaps that extend beyond 20-40 AU and a fraction of accreting disks above $12 \%$ at least, and possibly closer to $55 \%$ (see the models with $\alpha_{\mathrm{dz}}=1 \times 10^{-4}$ and $3 \times 10^{-4}$, for $r_{\mathrm{dz}}=20 \mathrm{AU}$, Table 2).

Finally, we note that our choice for $\alpha_{\mathrm{a}}$ underestimates the mean values found by the more complex model. A higher turbulence in the MRI-active layer could result in the photoevaporative gap to open earlier, which we expect to result in a higher dust content in the outer disk, without decreasing the fraction of accreting disks found in this work. Indeed, $\alpha_{\mathrm{dz}}$ and $r_{\mathrm{dz}}$ are the key parameters here since these determine the lifetime and viscous timescale of the inner disk.

\section{Summary}

In this work, we present a disk evolution model, which includes $\mathrm{X}$-ray photoevaporative dispersal and a dead-zone prescription, 
in order to explain the accretion rates and extended gap sizes observed in transition disks. Our population synthesis study shows that considering a dead zone in the inner regions can easily result in transition disks with extended gaps $\left(r_{\text {gap }} \gtrsim 20 \mathrm{AU}\right)$ and long-lived inner disks capable of sustaining high accretion rates $\left(\dot{M}_{\mathrm{g}} \sim 10^{-9} M_{\odot} \mathrm{yr}^{-1}\right)$ during the dispersal process. This is a result of the differential evolution of the inner and outer disk. Because the outer turbulent disk evolves on shorter viscous timescales, its accretion rate decreases faster and enters into the photoevaporative dispersal regime earlier. Meanwhile, the viscous timescale of the inner disk is longer due to the low turbulence in the dead zone, allowing it to sustain a relatively high accretion rate.

For dead-zone properties of $\alpha_{\mathrm{dz}}=10^{-4}$ and $r_{\mathrm{dz}}=10 \mathrm{AU}$, we predict that $63 \%$ of transition disks should still be accreting, with $\dot{M}_{\mathrm{g}} \gtrsim 10^{-11} M_{\odot} \mathrm{yr}^{-1}$. We also find that half of these accreting transition disks display high accretion rates of $\dot{M}_{\mathrm{g}} \gtrsim$ $5.0 \times 10^{-10} M_{\odot} \mathrm{yr}^{-1}$. This means that photoevaporative disk dispersal could, in fact, explain the observed accreting transition disks with large gaps, though it is still necessary to explain why we do not detect the predicted fraction of non-accreting disks.

While our model does not explicitly predict transition disks with narrow gaps ( $\left.r_{\mathrm{dz}} \lesssim 10 \mathrm{AU}\right)$, we suggest that these could still be explained through other processes, such as dust growth within a dead zone during the earlier stages of disk evolution, which would then evolve into wide photoevaporative gaps at later stages. From our dust-evolution simulations, we learn that the inner disk retains a dust component consisting of large millimeter-to-centimeter-sized grains, while the dust in the outer disk forms a ring at the edge of the photoevaporative gap, with grain sizes between one micrometer and a few millimeters. Radiative transfer calculations based on our dust distribution model show an inner and outer disk in the $1.3 \mathrm{~mm}$ continuum, with the inner disk being fainter by a factor of a few, and that the SED shows an emission deficit at NIR and MIR wavelengths, which is a characteristic feature of transition disk spectra.

While the millimeter flux in our model appears to be fainter than the ones observed in transition disks with large cavities, this could be solved by including dust traps in the outer disk (such as the ones caused by planets), or more vigorous photoevaporation rates (as those predicted for carbon depleted disks), in order to retain a higher fraction of solids during the early stages of disk evolution.

In future studies, we plan to focus on the observability of photoevaporating disks by extending our analysis of the dust component to determine if the predicted non-accreting disks could be too faint to be observed and if additional substructures could reproduce the observed population of millimeter bright transition disks with high accretion rates. In summary, our model including dead zones and photoevaporation is a good candidate to explain several features observed in transition disks, from the high accretion rates, to the large gaps, and even the inner disks observed in the millimeter continuum.

Acknowledgements. We would like to thank the anonymous referee for the useful feedback, that greatly improved the quality of this work. The authors acknowledge funding from the Alexander von Humboldt Foundation in the framework of the Sofja Kovalevskaja Award endowed by the Federal Ministry of Education and Research, from the European Research Council (ERC) under the European Union's Horizon 2020 research and innovation programme under grant agreement No 714769, and by the Deutsche Forschungsgemeinschaft (DFG, German Research Foundation) under Germany's Excellence Strategy - EXC2094 - 390783311 and Ref no. FOR 2634/1.

\section{References}

Alcalá, J. M., Natta, A., Manara, C. F., et al. 2014, A\&A, 561, A2 Alcalá, J. M., Manara, C. F., Natta, A., et al. 2017, A\&A, 600, A20 Alexander, R. D., \& Armitage, P. J. 2007, MNRAS, 375, 500

Alexander, R. D., Clarke, C. J., \& Pringle, J. E. 2006a, MNRAS, 369, 216 Alexander, R. D., Clarke, C. J., \& Pringle, J. E. 2006b, MNRAS, 369, 229 ALMA Partnership, (Brogan, C. L., et al.) 2015, ApJ, 808, L3 Andrews, S. M., Wilner, D. J., Zhu, Z., et al. 2016, ApJ, 820, L40 Andrews, S. M., Huang, J., Pérez, L. M., et al. 2018, ApJ, 869, L41 Asensio-Torres, R., Henning, T., Cantalloube, F., et al. 2021, A\&A 652, A101 Audard, M., Ábrahám, P., Dunham, M. M., et al. 2014, Protostars and Planets VI (Tucson: University of Arizona Press), 387

Bae, J., Hartmann, L., Zhu, Z., \& Gammie, C. 2013, ApJ, 774, 57 Balbus, S. A., \& Hawley, J. F. 1991, ApJ, 376, 214

Balbus, S. A., \& Hawley, J. F. 1998, Rev. Mod. Phys., 70, 1

Benisty, M., Tatulli, E., Ménard, F., \& Swain, M. R. 2010, A\&A, 511, A75

Birnstiel, T., Dullemond, C. P., \& Brauer, F. 2009, A\&A, 503, L5

Birnstiel, T., Dullemond, C. P., \& Brauer, F. 2010, A\&A, 513, A79

Birnstiel, T., Andrews, S. M., \& Ercolano, B. 2012a, A\&A, 544, A79

Birnstiel, T., Klahr, H., \& Ercolano, B. 2012b, A\&A, 539, A148

Birnstiel, T., Dullemond, C. P., Zhu, Z., et al. 2018, ApJ, 869, L45

Blandford, R. D., \& Payne, D. G. 1982, MNRAS, 199, 883

Booth, R. A., \& Clarke, C. J. 2021, MNRAS, 502, 1569

Brauer, F., Dullemond, C. P., \& Henning, T. 2008, A\&A, 480, 859

Christiaens, V., Cantalloube, F., Casassus, S., et al. 2019, ApJ, 877, L33

Cieza, L., Padgett, D. L., Stapelfeldt, K. R., et al. 2007, ApJ, 667, 308

Cieza, L. A., Schreiber, M. R., Romero, G. A., et al. 2010, ApJ, 712, 925

Cieza, L. A., Schreiber, M. R., Romero, G. A., et al. 2012, ApJ, 750, 157

Clarke, C. J., Gendrin, A., \& Sotomayor, M. 2001, MNRAS, 328, 485

Curran, R. L., Argiroffi, C., Sacco, G. G., et al. 2011, A\&A, 526, A104

Currie, T., \& Sicilia-Aguilar, A. 2011, ApJ, 732, 24

Donehew, B., \& Brittain, S. 2011, AJ, 141, 46

Draine, B. 2006, ApJ, 636, 1114

Dra̧żkowska, J., \& Alibert, Y. 2017, A\&A, 608, A92

Drążkowska, J., Li, S., Birnstiel, T., Stammler, S. M., \& Li, H. 2019, ApJ, 885, 91

Dubrulle, B., Morfill, G., \& Sterzik, M. 1995, Icarus, 114, 237

Dullemond, C. P., \& Dominik, C. 2005, A\&A, 434, 971

Dullemond, C. P., Juhasz, A., Pohl, A., et al. 2012, Astrophysics Source Code Library [record ascl: 1202.015]

Ercolano, B., \& Clarke, C. J. 2010, MNRAS, 402, 2735

Ercolano, B., \& Pascucci, I. 2017, Roy. Soc. Open Sci., 4, 170114

Ercolano, B., Drake, J. J., Raymond, J. C., \& Clarke, C. C. 2008, ApJ, 688, 398

Ercolano, B., Clarke, C. J., \& Drake, J. J. 2009, ApJ, 699, 1639

Ercolano, B., Weber, M. L., \& Owen, J. E. 2018, MNRAS, 473, L64

Espaillat, C., D’Alessio, P., Hernández, J., et al. 2010, ApJ, 717, 441

Espaillat, C., Muzerolle, J., Najita, J., et al. 2014, in Protostars and Planets VI, eds. H. Beuther, R. S. Klessen, C. P. Dullemond, \& T. Henning (Tucson: University of Arizona Press), 497

Flock, M., Ruge, J. P., Dzyurkevich, N., et al. 2015, A\&A, 574, A68

Flock, M., Turner, N. J., Nelson, R. P., et al. 2020, ApJ, 897, 155

Follette, K. B., Grady, C. A., Swearingen, J. R., et al. 2015, ApJ, 798, 132

Francis, L., \& van der Marel, N. 2020, ApJ, 892, 111

Franz, R., Picogna, G., Ercolano, B., \& Birnstiel, T. 2020, A\&A, 635, A53

Fromang, S., \& Nelson, R. P. 2009, A\&A, 496, 597

Gammie, C. F. 1996, ApJ, 457, 355

Gárate, M., Birnstiel, T., Stammler, S. M., \& Günther, H. M. 2019, ApJ, 871, 53 Gárate, M., Birnstiel, T., Dra̧żkowska, J., \& Stammler, S. M. 2020, A\&A, 635, A149

Garcia Lopez, R., Natta, A., Testi, L., \& Habart, E. 2006, A\&A, 459, 837

GRAVITY Collaboration (Perraut, K., et al.) 2019, A\&A, 632, A53

GRAVITY Collaboration (Bouarour, Y. I., et al.) 2020, A\&A, 642, A162

Güdel, M., Skinner, S. L., Mel'Nikov, S. Y., et al. 2007, A\&A, 468, 529

Gundlach, B., \& Blum, J. 2015, ApJ, 798, 34

Gundlach, B., Kilias, S., Beitz, E., \& Blum, J. 2011, Icarus, 214, 717

Hardy, A., Caceres, C., Schreiber, M. R., et al. 2015, A\&A, 583, A66

Hendler, N. P., Pinilla, P., Pascucci, I., et al. 2018, MNRAS, 475, L62

Henning, T., \& Stognienko, R. 1996, A\&A, 311, 291

Huang, J., Andrews, S. M., Cleeves, L. I., et al. 2018, ApJ, 852, 122

Hutchison, M. A., \& Clarke, C. J. 2021, MNRAS, 501, 1127

Hutchison, M. A., Price, D. J., Laibe, G., \& Maddison, S. T. 2016, MNRAS, 461, 742

Kataoka, A., Muto, T., Momose, M., et al. 2015, ApJ, 809, 78

Keane, J. T., Pascucci, I., Espaillat, C., et al. 2014, ApJ, 787, 153

Keppler, M., Benisty, M., Müller, A., et al. 2018, A\&A, 617, A44

Keppler, M., Teague, R., Bae, J., et al. 2019, A\&A, 625, A118

Kluska, J., Kraus, S., Davies, C. L., et al. 2018, ApJ, 855, 44 
Komaki, A., Nakatani, R., \& Yoshida, N. 2021, ApJ, 910, 51

Kretke, K. A., Lin, D. N. C., Garaud, P., \& Turner, N. J. 2009, ApJ, 690, 407

Kunitomo, M., Ida, S., Takeuchi, T., et al. 2021, ApJ, 909, 109

Lubow, S. H., \& D’Angelo, G. 2006, ApJ, 641, 526

Lüst, R. 1952, Zeitschrift Naturforschung Teil A, 7, 87

Lynden-Bell, D., \& Pringle, J. E. 1974, MNRAS, 168, 603

Manara, C. F., Testi, L., Natta, A., et al. 2014, A\&A, 568, A18

Manara, C. F., Fedele, D., Herczeg, G. J., \& Teixeira, P. S. 2016a, A\&A, 585, A136

Manara, C. F., Rosotti, G., Testi, L., et al. 2016b, A\&A, 591, L3

Manara, C. F., Testi, L., Herczeg, G. J., et al. 2017, A\&A, 604, A127

Manger, N., Klahr, H., Kley, W., \& Flock, M. 2020, MNRAS, 499, 1841

Martin, R. G., \& Lubow, S. H. 2011, ApJ, 740, L6

Mathis, J. S., Rumpl, W., \& Nordsieck, K. H. 1977, ApJ, 217, 425

Matter, A., Labadie, L., Augereau, J. C., et al. 2016, A\&A, 586, A11

Merín, B., Brown, J. M., Oliveira, I., et al. 2010, ApJ, 718, 1200

Morishima, R. 2012, MNRAS, 420, 2851

Najita, J. R., Strom, S. E., \& Muzerolle, J. 2007, MNRAS, 378, 369

Nakagawa, Y., Sekiya, M., \& Hayashi, C. 1986, Icarus, 67, 375

Nelson, R. P., Gressel, O., \& Umurhan, O. M. 2013, MNRAS, 435, 2610

Olofsson, J., Benisty, M., Le Bouquin, J. B., et al. 2013, A\&A, 552, A4

Ormel, C. W., \& Cuzzi, J. N. 2007, A\&A, 466, 413

Owen, J. E. 2016, PASA, 33, e005

Owen, J. E., \& Kollmeier, J. A. 2019, MNRAS, 487, 3702

Owen, J. E., Ercolano, B., Clarke, C. J., \& Alexander, R. D. 2010, MNRAS, 401, 1415

Owen, J. E., Ercolano, B., \& Clarke, C. J. 2011, MNRAS, 412, 13

Owen, J. E., Clarke, C. J., \& Ercolano, B. 2012, MNRAS, 422, 1880

Owen, J. E., Hudoba de Badyn, M., Clarke, C. J., \& Robins, L. 2013, MNRAS, 436, 1430

Pascucci, I., Hollenbach, D., Najita, J., et al. 2007, ApJ, 663, 383

Picogna, G., Ercolano, B., Owen, J. E., \& Weber, M. L. 2019, MNRAS, 487, 691

Pinilla, P., Benisty, M., \& Birnstiel, T. 2012a, A\&A, 545, A81

Pinilla, P., Birnstiel, T., Ricci, L., et al. 2012b, A\&A, 538, A114

Pinilla, P., de Juan Ovelar, M., Ataiee, S., et al. 2015, A\&A, 573, A9

Pinilla, P., Flock, M., Ovelar, M. d. J., \& Birnstiel, T. 2016a, A\&A, 596, A81

Pinilla, P., Klarmann, L., Birnstiel, T., et al. 2016b, A\&A, 585, A35

Pinilla, P., Benisty, M., Cazzoletti, P., et al. 2019, ApJ, 878, 16

Pinilla, P., Pascucci, I., \& Marino, S. 2020, A\&A, 635, A105
Pinilla, P., Kurtovic, N. T., Benisty, M., et al. 2021, A\&A 649, A122 Powell, D., Murray-Clay, R., \& Schlichting, H. E. 2017, ApJ, 840, 93 Preibisch, T., Kim, Y.-C., Favata, F., et al. 2005, ApJS, 160, 401 Pringle, J. E. 1981, ARA\&A, 19, 137

Regály, Z., Juhász, A., Sándor, Z., \& Dullemond, C. P. 2012, MNRAS, 419, 1701

Romero, G. A., Schreiber, M. R., Cieza, L. A., et al. 2012, ApJ, 749, 79

Rosotti, G. P., Benisty, M., Juhász, A., et al. 2020, MNRAS, 491, 1335

Shakura, N. I., \& Sunyaev, R. A. 1973, A\&A, 24, 337

Skrutskie, M. F., Dutkevitch, D., Strom, S. E., et al. 1990, AJ, 99, 1187

Spezzi, L., Alcalá, J. M., Covino, E., et al. 2008, ApJ, 680, 1295

Strom, K. M., Strom, S. E., Edwards, S., Cabrit, S., \& Skrutskie, M. F. 1989, AJ, 97, 1451

Suzuki, T. K., Ogihara, M., Morbidelli, A., Crida, A., \& Guillot, T. 2016, A\&A, 596, A74

Takeuchi, T., \& Lin, D. N. C. 2002, ApJ, 581, 1344

Tripathi, A., Andrews, S. M., Birnstiel, T., \& Wilner, D. J. 2017, ApJ, 845, 44

Ueda, T., Flock, M., \& Okuzumi, S. 2019, ApJ, 871, 10

van Boekel, R., Henning, T., Menu, J., et al. 2017, ApJ, 837, 132

van der Marel, N., van Dishoeck, E. F., Bruderer, S., et al. 2016a, A\&A, 585, A58

van der Marel, N., Verhaar, B. W., van Terwisga, S., et al. 2016b, A\&A, 592, A126

Villenave, M., Benisty, M., Dent, W. R. F., et al. 2019, A\&A, 624, A7

Villenave, M., Ménard, F., Dent, W. R. F., et al. 2020, A\&A, 642, A164

Wada, K., Tanaka, H., Suyama, T., Kimura, H., \& Yamamoto, T. 2011, ApJ, 737, 36

Wang, L., \& Goodman, J. J. 2017, ApJ, 835, 59

Warren, S. G., \& Brandt, R. E. 2008, J. Geophys. Res. Atm., 113, D14220

Weber, P., Benítez-Llambay, P., Gressel, O., Krapp, L., \& Pessah, M. E. 2018 ApJ, 854, 153

Weidenschilling, S. J. 1977, MNRAS, 180, 57

Whipple, F. L. 1972, in From Plasma to Planet, ed. A. Elvius (Hoboken: John Wiley \& Sons ), 211

Wölfer, L., Picogna, G., Ercolano, B., \& van Dishoeck, E. F. 2019, MNRAS, 490, 5596

Youdin, A. N., \& Lithwick, Y. 2007, Icarus, 192, 588

Zhu, Z., Nelson, R. P., Hartmann, L., Espaillat, C., \& Calvet, N. 2011, ApJ, 729 47 
Table A.1. Percentage of transition disks with $\dot{M}_{g}>10^{-11} \mathrm{M}_{\odot} \mathrm{yr}^{-1}$ for different dead-zone models, using the initial condition in Equation A.1.

\begin{tabular}{ccc}
\hline \hline$\alpha_{\mathrm{dz}}$ & $r_{\mathrm{dz}}(\mathrm{AU})$ & Accreting disk fraction $(\%)$ \\
\hline \multirow{3}{*}{$10^{-4}$} & 20 & 100.0 \\
& 10 & 99.7 \\
& 5 & 52.8 \\
\hline \multirow{3}{*}{$3 \times 10^{-4}$} & 20 & 35.1 \\
& 10 & 25.0 \\
& 5 & 10.9 \\
\hline \multirow{3}{*}{$5 \times 10^{-4}$} & 20 & 4.7 \\
& 10 & 6.1 \\
& 5 & 3.2 \\
\hline
\end{tabular}

\section{Appendix A: Models in quasi-steady state}

In this appendix, we show an additional set of population synthesis models with a different initial condition, to complement the results of Section 4. For this work, we studied disks that are initialized with a Lynden-Bell \& Pringle (1974) profile, which consist of a power law with an exponential decay. When the turbulence profile is constant, these disks evolve in a "quasi-steady state", which is characterized by a constant accretion rate in the inner regions that decays uniformly over the viscous timescale. However, if a disk has a nonuniform turbulence profile, such as the one created by a dead zone (see Figure 2), this results in a variable accretion profile that is not in local equilibrium (see the initial condition of Figure 4, top panel). In order to test the effect of the initial condition, we repeated our population synthesis study as described in Section 3.2, but assuming the material is initially distributed such that the inner regions evolve in quasisteady state and using a scaled version of the Lynden-Bell \& Pringle (1974) profile:

$\Sigma_{\mathrm{g}}(r)=\Sigma_{0}\left(\frac{r}{r_{\mathrm{c}}}\right)^{-1} \exp \left(-r / r_{\mathrm{c}}\right) \frac{\alpha_{\mathrm{a}}}{\alpha(r)}$.

The scaling factor $\alpha_{\mathrm{a}} / \alpha(r)$ results in a profile that has a higher density in the dead-zone region and a lower density in the outer regions, when compared to the standard Lynden-Bell \& Pringle (1974) solution. The normalization factor $\Sigma_{0}$ is again defined such that the integrated mass is $M_{\text {disk }}=1 \mathrm{M}_{\odot}$.

Figure A.1 shows the corresponding distribution of the gas accretion rate and gap sizes of transition disks, and Table A.1 shows the fraction accreting transition disks using the initial condition from Equation A.1. From these new results, we immediately notice an increase in the abundance of transition disks accreting at high accretion rates. In particular, for the model with $\alpha_{\mathrm{dz}}=10^{-4}$ and $r_{\mathrm{dz}}=10 \mathrm{AU}$, we find that the predicted fraction of accreting transition disks jumped from $62.9 \%$ to $99.7 \%$, with approximately half of those disks displaying accretion rates above $10^{-9} \mathrm{M}_{\odot} \mathrm{yr}^{-1}$. Another difference is that now, using the scaled initial condition, the populations with more extended dead zones $\left(r_{\mathrm{dz}}=20 \mathrm{AU}\right)$ tend to display the highest fraction of accreting transition disks (for $\alpha_{\mathrm{dz}} \leq 3 \times 10^{-4}$ ).

Both the overall increment in the fraction of accreting transition disks and its systematic increase with the dead-zone size $r_{\mathrm{dz}}$, can be explained if we consider that the new initial condition represents the disk in which the inner regions are "saturated" of material; that is, they have the required amount of gas to be in local steady state. In this scenario, the inner disk has the maximum possible mass by the time photoevaporation opens a gap, and therefore it can survive for the longest possible time.
Of course, the question now would be if the quasi-steady state initial condition described by Equation A.1 can be met. From our results in Figure 3, we see that in the presence of a dead zone, a disk initialized with a Lynden-Bell \& Pringle (1974) profile slowly evolves into a quasi-steady state profile, like the one described by Equation A.1 (though the exact shape might vary), but with a lower normalization factor $\Sigma_{0}$ and larger characteristic radius $r_{\mathrm{c}}$, since some material must have been accreted, and the outer regions have expanded due to the transport of angular momentum.

We expect that disks with short viscous timescales in their inner regions would reach the quasi-steady state solution more easily. Accordingly, we find that the disk populations with smaller dead zones and higher turbulence parameters are less affected by the choice of initial condition (see Table 2 and Table A.1), since they have enough time to reach the quasi-steady state regime before the photoevaporation opens a gap and disconnects the inner and outer disk from each other. In contrast, disks with extended dead zones are more affected by the choice of initial condition, since their viscous timescales are longer (of several million years).

Another factor that should affect whether a disk reaches the quasi-state state solution is the disk mass, since a more massive disk would be able to transport more material into the inner regions before photoevaporation becomes relevant for its global evolution, though the corresponding normalization factor would be lower, depending on how much material was accreted into the star.

Finally, one reason why it might be preferable to draw our conclusions from the standard Lynden-Bell \& Pringle (1974) profile, instead of from the quasi-steady state profile in Equation A.1, is that the dead zones could be subject to thermal and gravitational instabilities during the early stages of disk evolution (Martin \& Lubow 2011). At that point, the turbulence is cyclically reactivated in the inner regions, resulting in accretion outbursts and the possibility of accumulated material being quickly flushed into the star (Kretke et al. 2009; Audard et al. 2014; Gárate et al. 2019). With this point in mind, we can think of the results based on the quasi-steady state initial condition as an upper limit to the fraction of accreting transition disks.

To conclude, we also show the effect of the quasi-steady state initial condition on the dust distribution and millimeter continuum synthetic observations in Figure A.2 and Figure A.3. As in Section 5, we used an X-ray luminosity of $L_{x}=10^{31} \mathrm{ergs}^{-1}$, $\alpha_{\mathrm{dz}}=10^{-4}$, and $r_{\mathrm{dz}}=10 \mathrm{AU}$, but we take the snapshot at 1.2 Myr, since the gap opens earlier when using the initial condition from Equation A.1. The main difference is that now the surface brightness of the inner disk is comparable to that of the outer ring in the millimeter continuum, as observed in some transition disks (Hendler et al. 2018; Pinilla et al. 2021). 

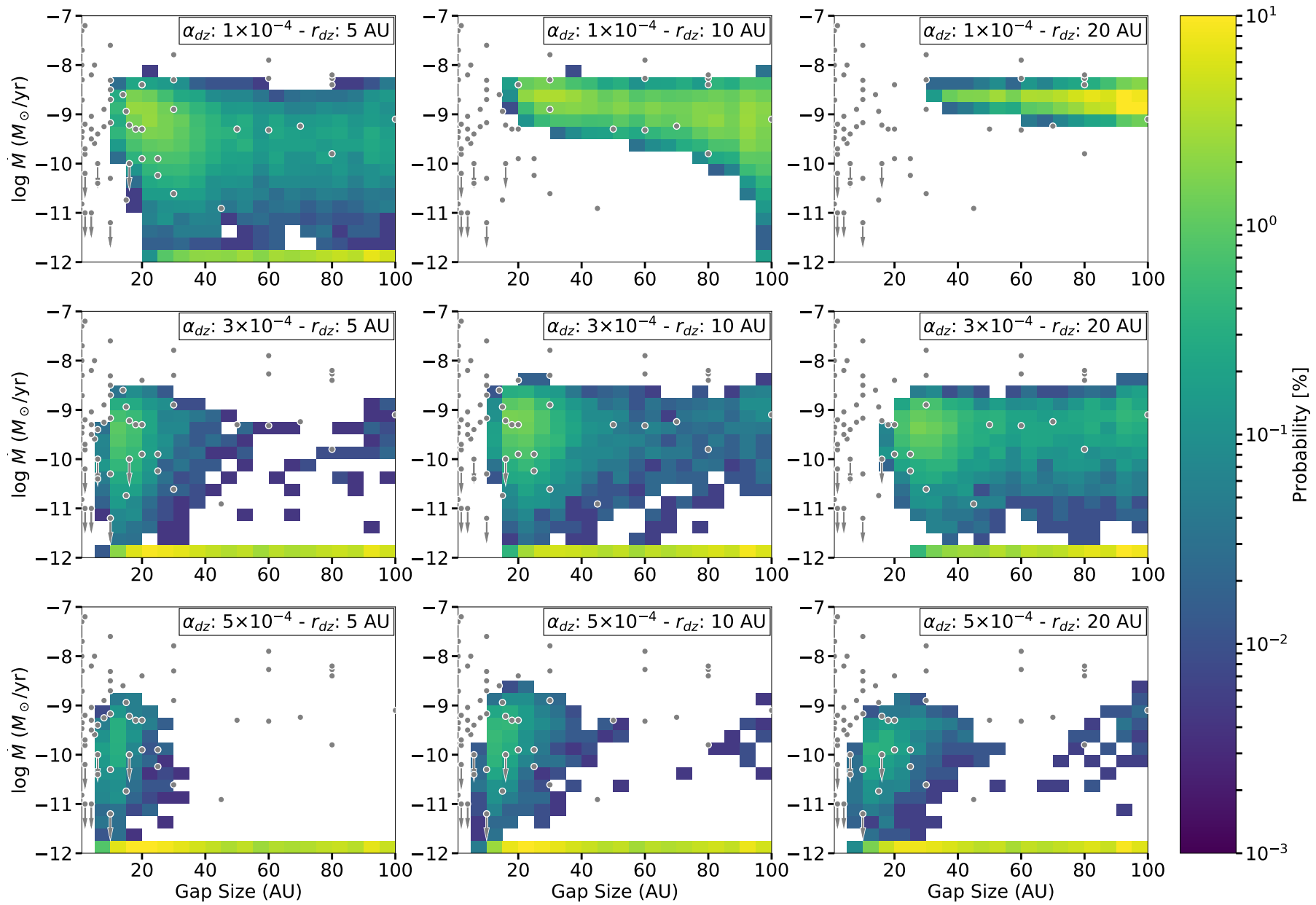

Fig. A.1. Same as Figure 7, but using the initial condition described in Equation A.1.

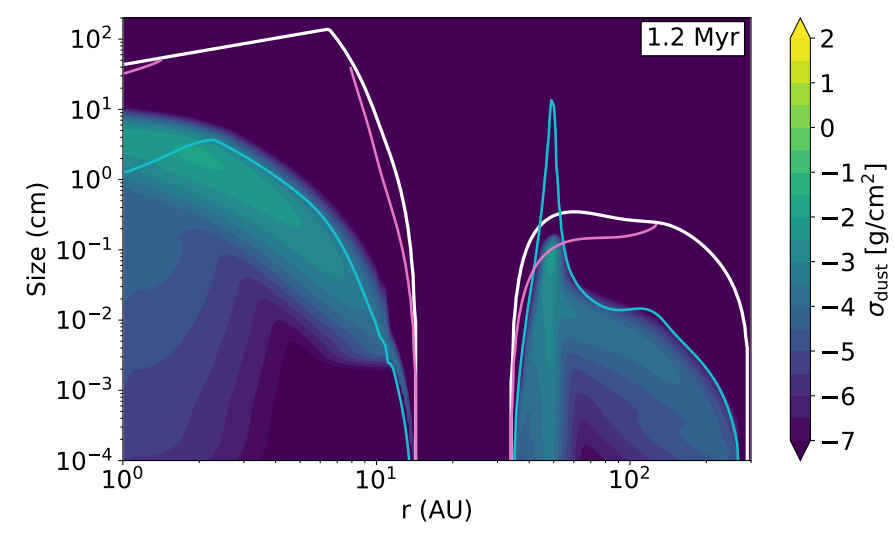

Fig. A.2. Dust size distribution at $1.2 \mathrm{Myr}$ for a disk evolution model initialized in quasi-steady state (Equation A.1). All the other parameters are the same as in Figure 9.

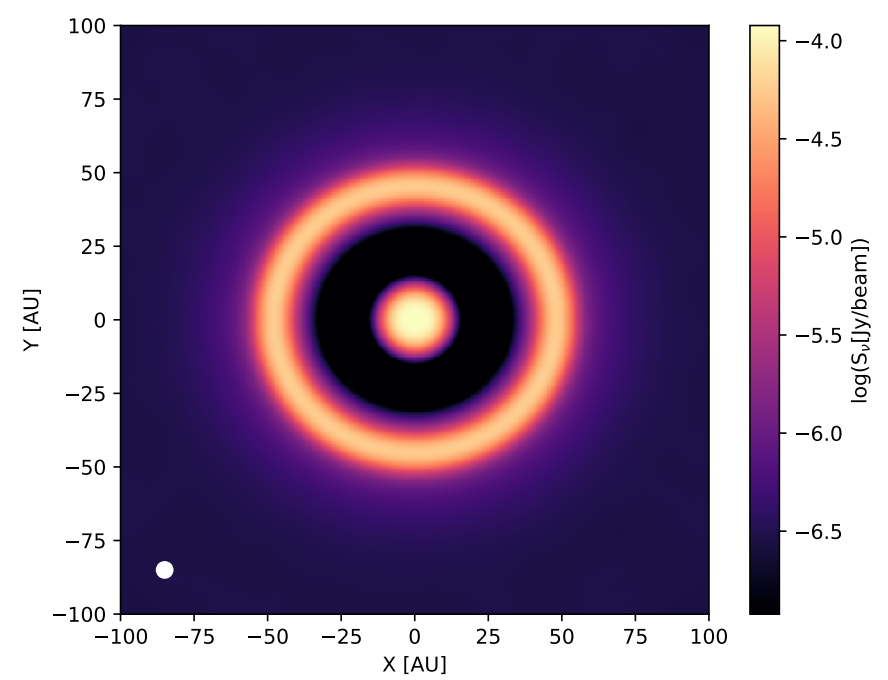

Fig. A.3. Radiative transfer model of the continuum emission at $\lambda=$ $1.3 \mathrm{~mm}$, for the dust size distribution presented in Figure A.2 and convolved with a Gaussian beam of 40 mas. The beam size is shown in the lower left corner. 